\title{
Detection of vibrationally excited methyl formate in W51 e2
}

\author{
K. Demyk ${ }^{1, \star}$, G. Wlodarczak ${ }^{1}$, and M. Carvajal ${ }^{2}$ \\ ${ }^{1}$ Laboratoire de Physique des Lasers, Atomes et Molécules, UMR CNRS 8523 Université Lille 1, 59655 Villeneuve d'Ascq Cedex, \\ France \\ e-mail: Karine.demyk@cesr.fr \\ 2 Departamento de Fisica Aplicada, Facultad de Ciencias Experimentales, Universidad de Huelva, 21071 Huelva, Spain
}

Received 4 January 2008 / Accepted 30 June 2008

\begin{abstract}
Context. Hot cores in molecular clouds, such as Orion KL, Sgr B2, W51 e1/e2, are characterized by the presence of molecules at sufficiently high temperatures to populate their low-frequency vibrationally excited states significantly. Complex organic molecules, such as methyl formate, ethyl cyanide or dimethyl ether, are characterized by a dense spectrum both in the ground state and in the excited states and lines from vibrationally excited states certainly participate to the spectral confusion.

Aims. Following a laboratory study of the first torsional excited mode of methyl formate, we search for methyl formate, $\mathrm{HCOOCH}_{3}$, in its first torsionally excited state $\left(v_{\mathrm{t}}=1\right)$ in the molecular cloud W51 e2.

Methods. We performed observations of the molecular cloud W51 e2 in different spectral regions at 1.3, 2, and $3 \mathrm{~mm}$ with the IRAM 30 m single dish antenna.

Results. Methyl formate in its first torsionally excited state $\left(v_{\mathrm{t}}=1\right.$ at $\left.131 \mathrm{~cm}^{-1}\right)$ is detected for the first time toward W51 e2. We detect 82 transitions among which 46 are unblended with other species. For a total of 16 A-E pairs in the observed spectrum, 9 are unblended; these 9 pairs are all detected. All transitions from excited methyl formate within the observed spectral range are detected and no strong lines are missing. The column density of the excited state is comparable to that of the ground state. For a source size of 7", we find that $T_{\text {rot }}=104 \pm 14 \mathrm{~K}$ and $N=9.4_{-2.8}^{+4.0} \times 10^{16} \mathrm{~cm}^{-2}$ for the excited state and $T_{\text {rot }}=176 \pm 24 \mathrm{~K}$ and $N=1.7_{-.2}^{+2} \times 10^{17} \mathrm{~cm}^{-2}$ for the ground state. Lines from ethyl cyanide in its two first excited states $\left(v_{\mathrm{t}}=1\right.$, torsion mode at $\left.212 \mathrm{~cm}^{-1}\right)$ and $\left(v_{\mathrm{b}}=1, \mathrm{CCN}\right.$ in-plane bending mode at $206 \mathrm{~cm}^{-1}$ ) are also present in the observed spectrum. Blending problems prevent a precise estimate of its abundance, although as for methyl formate, it should be comparable to the value derived for the ground state for which we find $T_{\text {rot }}=103 \pm 9 \mathrm{~K}$ and $N=3.7_{-04}^{+0.6} \times 10^{15} \mathrm{~cm}^{-2}$ for a $7^{\prime \prime}$ source size.

Conclusions. With regard to the number of lines of excited methyl formate and ethyl cyanide detected in W51 e2, it appears that excited states of large molecules certainly account for a significant number of unidentified lines in spectral survey of molecular clouds.
\end{abstract}

Key words. ISM: molecules - ISM: abundances - ISM: individual objects: W51 e2 - radio lines: ISM - line: identification methods: observational

\section{Introduction}

W51 e2 is a hot core part of the $\mathrm{W} 51 \mathrm{H}_{\mathrm{II}}$ region located in the Sagittarius spiral arm at a distance of about 7-8 kpc. It is a region of high-mass star formation. W51 e2 and W51 e1 appear to be important star-forming cores. They exhibit a rich chemistry, comparable to that observed either in Orion or Sagittarius. Numerous large organic molecules have been observed in their direction. $\mathrm{CH}_{3} \mathrm{CN}$ and $\mathrm{CS}$ maps were studied by Zhang et al. (1998). Formic acid (HCOOH) was mapped by Liu et al. (2001). Methyl formate $\left(\mathrm{HCOOCH}_{3}\right)$ and ethyl cyanide $\left(\mathrm{CH}_{3} \mathrm{CH}_{2} \mathrm{CN}\right.$ ) were observed in several studies (Liu et al. 2001; Ikeda et al. 2001; Remijan et al. 2002). Ikeda et al. (2001) studied ethylene oxide $\left(\mathrm{c}-\mathrm{C}_{2} \mathrm{H}_{4} \mathrm{O}\right)$ and its isomer acetaldehyde $\left(\mathrm{CH}_{3} \mathrm{CHO}\right)$. Acetic acid $\left(\mathrm{CH}_{3} \mathrm{COOH}\right)$ was detected by Remijan et al. (2002) with a fractional abundance of $(1-6) \times 10^{-2}$ relative to $\mathrm{HCOOCH}_{3}$. Glycine, whose presence may be inferred from the observations of acetic acid with which it shares common structural elements, was not detected in W51 e2 (Snyder et al. 2005). Trans-ethyl methyl ether was however detected in W51 e2 (Fuchs et al. 2005).

\footnotetext{
* Present address: Centre d'Étude Spatiale des Rayonnements, Université Paul Sabatier, 31028 Toulouse, France.
}

The rotational temperature of most of these large molecular species is high. Liu et al. (2001) estimated the rotational temperature in W51 e2 to be in the range 200-300 K. From high resolution observations of $\mathrm{CH}_{3} \mathrm{CN}$ analyzed with statistical equilibrium models, Remijan et al. (2004) derived the kinetic temperature in $\mathrm{W} 51 \mathrm{e} 2$ to be $T_{\text {kin }}=153(21) \mathrm{K}$. At such temperature, low-energy vibrational excited states can be significantly populated. Transitions from vibrationally excited states have indeed been observed in other sources such as in Sgr B2(N-LMH) for $\mathrm{C}_{2} \mathrm{H}_{3} \mathrm{CN}, \mathrm{CH}_{3} \mathrm{CH}_{2} \mathrm{OH}$ (Nummelin et al. 1998) and $\mathrm{CH}_{3} \mathrm{CH}_{2} \mathrm{CN}$ (Mehringer et al. 2004). Lines from torsionally excited methyl formate have been identified in Orion KL (Kobayashi et al. 2007).

In this study, we present the first detection of excited methyl formate and ethyl cyanide in the molecular cloud W51 e2. Our objective was to detect methyl carbamate $\left(\mathrm{NH}_{2} \mathrm{COOCH}_{3}\right)$, an isomer of glycine $\left(\mathrm{NH}_{2} \mathrm{CH}_{2} \mathrm{COOH}\right)$, which has a larger dipole moment, making its detection more favorable than glycine. It was not detected. However, a few strong unidentified lines in the data attracted our attention and were attributed to methyl formate in its first torsional excited state. Further observations confirmed this assertion and lead to the detection of excited ethyl cyanide in W51 e2. The first vibrationally excited state of methyl formate 
is the $\mathrm{CH}_{3}$ torsion mode, $v_{18}$, hereafter referred to as $v_{\mathrm{t}}=1$, at $131 \mathrm{~cm}^{-1}(188 \mathrm{~K})$. The rotational spectrum in this excited state was measured and analyzed by Ogata et al. (2004). Ethyl cyanide has two close vibrationally excited states, the CCN inplane bending mode, $v_{13}$, hereafter called $v_{\mathrm{b}}=1$, at $206 \mathrm{~cm}^{-1}$ $(296 \mathrm{~K})$ and the $\mathrm{CH}_{3}$ torsion mode, $v_{21}$, hereafter called $v_{\mathrm{t}}=1$, at $212 \mathrm{~cm}^{-1}(305 \mathrm{~K})$. A preliminary analysis of the rotational spectrum in these two excited states is presented by Mehringer et al. (2004).

The observations and methods used for the data analysis are described in Sects. 2 and 3, respectively. The study of methyl formate and ethyl cyanide in the ground and excited state is presented in Sects. 4 and 5, respectively. The search for methyl carbamate and glycine is presented in Sect. 6. Our discussion is completed in Sect. 7.

\section{Observations}

The observations were performed with the IRAM $30 \mathrm{~m}$ antenna at Pico Veleta (Spain) in June 2003 and June 2006. W51 e2 was observed at the position $\alpha(2000)=19^{\circ} 23^{\prime} 43.9^{\prime \prime}$ and $\delta(2000)=14^{\circ} 30^{\prime} 34.8^{\prime \prime}$ in position switching mode with the OFF position located at $\alpha=300^{\prime \prime}$ and $\delta=0^{\prime \prime}$.

Several spectral windows in the $80-250 \mathrm{GHz}$ range were observed to include as many transitions as possible for the searched molecules (excited methyl formate and ethyl cyanide for the 2006 data and methyl carbamate and glycine for the 2003 data) and as few transitions as possible of other molecules with numerous strong lines (such as methyl formate and ethyl cyanide in the ground state and dimethyl ether).

All lines were observed with an array of 4 receivers (in single-side band mode) set at the appropriate frequencies. The spectrometers used were a low resolution $1 \mathrm{MHz}$ filter bank and an autocorrelator with a spectral resolution in the $40-320 \mathrm{kHz}$ range, split between different receivers. Focus and pointing were checked regularly by observing the nearby ultra compact HII region $\mathrm{K} \mathrm{3-50A}$. The rejection of the image band (USB) was about $26 \mathrm{~dB}$ at $3 \mathrm{~mm}, 12 \mathrm{~dB}$ at $2 \mathrm{~mm}, 15 \mathrm{~dB}$ at $1.3 \mathrm{~mm}$, and $10 \mathrm{~dB}$ at $1.1 \mathrm{~mm}$. The system temperature was typically 100 $200 \mathrm{~K}, 200-700 \mathrm{~K}, 200-700 \mathrm{~K}$, and $400-1500 \mathrm{~K}$ at 3, 2, 1.3, and $1.1 \mathrm{~mm}$, respectively. The total usable $\mathrm{ON}+\mathrm{OFF}$ integration time varied from 30 to 50 min depending on the frequency range. The beam size was $22^{\prime \prime}, 17^{\prime \prime}$, and $10.5^{\prime \prime}$ at 3,2 , and $1.3 \mathrm{~mm}$, respectively. The spectra are presented in main beam temperature unit, which is calculated from the antenna temperature: $T_{\mathrm{mb}}=F_{\mathrm{eff}} / B_{\mathrm{eff}} \times T_{\mathrm{A}}^{*}$. The data were reduced using the GILDAS package.

\section{Analysis}

For the data analysis, we assume that local thermodynamic equilibrium (LTE) is reached, i.e. we assume that the excitation, rotational, and vibrational temperatures are equal to the kinetic temperature in the emitting region and that the lines are thermalized, i.e. their level population is described by a Boltzmann distribution at that temperature. The validity of this assumption is discussed in Sect. 7.

The data were analyzed using the classical rotational diagram method to estimate the rotational temperature and the column density with their uncertainties for the different identified species. We adopted the formulation from Turner (1991), corrected for beam dilution effects:

$\ln \left(\frac{3 k W}{8 \pi^{3} v S \mu^{2} g_{i} g_{k}}\right)=\ln \left(\frac{N}{Q}\right)-\frac{E_{\mathrm{u}}}{k T}-\ln (b)$ where $W$ is the integrated line intensity in $\mathrm{K} \mathrm{km} \mathrm{s}^{-1}, v$ the line frequency, $S \mu^{2}$ the line strength in Debye ${ }^{2}, g_{i}$ the reduced nuclear spin statistical weight, $g_{k}$ the $K$-level degeneracy, $Q$ is the partition function, $E_{\mathrm{u}}$ the upper state energy, $N$ is the total column density, and $T$ is the excitation temperature. Assuming a Gaussian beam, the beam dilution factor $b$ is given by:

$b=\frac{\theta_{\mathrm{s}}^{2}}{\theta_{\mathrm{s}}^{2}+\theta_{\mathrm{tel}}^{2}}$

where $\theta_{\mathrm{s}}$ and $\theta_{\text {tel }}$ are the source and telescope beam size in arcsecond, respectively.

Beam dilution effects were taken into account both in the rotational diagram analysis and in the emission modeling (see below). The emission region in W51 e2 was observed to be smaller than $10^{\prime \prime}$ for most organic molecules (Remijan et al. 2002; Liu et al. 2001). Consequently beam dilution effects were important at low frequency at which the IRAM $30 \mathrm{~m}$ antenna beam size is significantly larger $\left(29^{\prime \prime}\right.$ at $\left.86 \mathrm{GHz}\right)$.

We compared the observed spectrum with simulated spectra calculated using a simple emission model at local thermodynamic equilibrium (LTE). The expression for the simulated main beam temperature for one molecule was thus:

$T_{\mathrm{mb}}=b \times\left(J-J_{\mathrm{bg}}\right) \times\left(1-\mathrm{e}^{-\tau}\right)$

where $J$ is the source function:

$J=\frac{h v}{k} \times\left(\mathrm{e}^{h v / k T}-1\right)^{-1}$

and

$J_{\text {bg }}=\frac{h v}{k} \times\left(\mathrm{e}^{h v / k \times 2.7}-1\right)^{-1}$

$\tau$ is the optical depth, summed over all the transitions of the molecules:

$\tau=\sum_{i} \frac{c^{2}}{8 \pi v^{2}} N_{\mathrm{tot}} \frac{g_{\mathrm{u}}}{Q} A_{\mathrm{ul}} \Phi(v) \mathrm{e}^{-E_{l} / k T}\left(1-\mathrm{e}^{-E_{\mathrm{ul}} / k T}\right)$

and $\Phi(v)$ is the line profile:

$\Phi(v)=\frac{1}{\sqrt{\pi \Delta v_{\mathrm{D}}}} \times \mathrm{e}^{-\left(v-v_{i}\right)^{2} / \Delta v_{\mathrm{D}}^{2}}$

$A_{\mathrm{ul}}$ is the Einstein coefficient, $E_{\mathrm{ul}}$ the energy of the transition, $E_{\mathrm{l}}$ the energy of the lower state, $g_{\mathrm{u}}$ is the upper state degeneracy, $Q$ the partition function, $\Delta \nu_{\mathrm{D}}$ is the Doppler width of the line, $b$ is the beam dilution correction factor, and $N_{\text {tot }}$ is the total column density.

In hot cores, the temperature is such that the low-energy vibrational and/or torsional excited modes are significantly populated. We therefore used the vibrational-rotational partition function, $Q_{\mathrm{rv}}$, instead of the pure rotational partition function. Assuming non-interacting harmonic vibrational levels and rigid rotor levels, the ro-vibrational partition function was approximated by (see Gordy \& Cook 1984):

$Q_{\mathrm{rv}}=\prod_{i}\left(1-\mathrm{e}^{-h v_{i} / k T}\right)^{-d_{i}} \times Q_{\mathrm{rot}}$

where $v_{i}$ is the frequency of the vibrational mode $i, d_{i}$ its degeneracy, and $Q_{\text {rot }}$ is the rotational partition function. $Q_{\text {rot }}$ is approximated by:

$Q_{\mathrm{rot}}=\sigma \times \sqrt{\frac{\pi(k T)^{3}}{h^{3} A B C}}$ 
where $\sigma$ is the symmetry number (see Gordy \& Cook 1984). At LTE, the temperature used to calculate the rotational and vibrational partition function was the same: we therefore assumed implicitly that $T_{\text {vib }}=T_{\text {rot }}$, hypothesis that may not be valid (see Sect. 7). For methyl formate that has one internal rotor, rotational transitions are split into $\mathrm{A}$ and $\mathrm{E}$ components and $\sigma$ is equal to 2. To calculate the partition function, we considered the first excited state of methyl formate at $131 \mathrm{~cm}^{-1}$. For ethyl cyanide, we used the partition function given by Mehringer et al. (2004), which is calculated by summing the rotational states of the ground state and the first two excited states of ethyl cyanide at 206 and $212 \mathrm{~cm}^{-1}$. For methyl carbamate, we used the partition function in the ground vibrational state given by Groner et al. (2007).

\section{Torsionally excited methyl formate}

The predictions for the methyl formate lines are based on the work on this species by Carvajal et al. (2007). In this work, all experimental data available on the ground and excited states (3496 and 774 microwave lines, respectively) in the 7-200 GHz frequency range, covering the $J$ values up to 43 in the ground state and up to 18 in the first excited state $v_{\mathrm{t}}=1$, were collected from previously published studies (Ogata et al. 2004; Oesterling et al. 1999; Plummer et al. 1986, 1984; Demaison et al. 1984). Carvajal et al. (2007) also added 434 new lines of methyl formate in the ground state, measured in Lille in the $567-669 \mathrm{GHz}$ spectral range and corresponding to transitions with $J$ and $K$ values of up to 62 and 22, respectively. This dataset was fitted with almost experimental accuracy (root-mean-square deviations of $94 \mathrm{kHz}$ and $84 \mathrm{kHz}$ for the 3496 (774) lines of the ground torsional state and of the excited state $v_{\mathrm{t}}=1$, respectively) using the so-called rho axis method (RAM) described in the literature (Hougen et al. 1994) and a model extended to include perturbation terms through to the eighth order. The spectroscopic parameters and the details of the fitting procedure are given by Carvajal et al. (2007) in which a table presenting all the fitted experimental frequencies, measurement uncertainties, calculated frequencies, observed-calculated values, line strengths, energy levels as well as identifications of the transitions, is available as Supplementary data.

For the detection of lines in the W51 e2 spectrum, we provided a line-list of predicted line-center frequencies and line intensities based on an internal rotation model (RAM or Rho Axis Method). This method and the code ${ }^{1}$ developed was used for several molecules detected in the interstellar medium: acetaldehyde, $\mathrm{CH}_{3} \mathrm{COH}$, (Kleiner et al. 1996), acetamide $\mathrm{CH}_{3} \mathrm{CONH}_{2}$, (Hollis et al. 2006), and acetic acid $\mathrm{CH}_{3} \mathrm{COOH}$, (Ilyushin et al. 2008). The laboratory measurements and predicted line frequencies of transitions in the first excited torsional state $v_{\mathrm{t}}=1$ of methyl formate in the spectral range used for the present detection are presented in Table 1 with the line assignment, the observed-calculated value, the experimental accuracy, the calculated uncertainty, the line strength, and the energy of the lower level ${ }^{2}$.

More than eighty transitions from torsionally excited methyl formate, 82 precisely, are detected in the source among which 46

\footnotetext{
${ }^{1}$ A version of the program is available at the web site (http://www . ifpan.edu.pl/ kisiel/introt/introt.htm\#belgi) and other versions are available from Kleiner (see the web site for more information).

2 A prediction of the overall spectrum of methyl formate in the ground and first excited states, for a large frequency range, will be published soon (Kleiner et al. in preparation).
}

are not blended. Taking into account possible blending, we find that all lines from torsionally excited methyl formate, predicted to be sufficiently intense to be detectable, are present in the observed spectra. Furthermore, no lines, such as unobserved strong methyl formate lines, contradicts the identification in the observed spectral range. The internal rotation of the methyl group of methyl formate splits the transitions into A and E components of the same intensity. Among the 16 observed A-E pairs, 9 are not blended (see Fig. 1). The intensity of the lines in a A-E pair, when no lines of the pair is blended, is consistent with the expected ones, strengthening the identification of excited methyl formate in this source. The detected lines (observed frequency, integrated intensity, and line intensity) are listed in Table 2 with the laboratory or calculated frequency, the quantum numbers of the transition, its line strength, and lower state energy. The first column of Table 2 indicates the line number used to label the lines in Fig. 1. The number in parenthesis indicates the A or E line associated with the transition when it is observed. However, about half of the lines are blended. Comments were added in Table 2 to indicate line blenders when they are identified.

The non-blended lines were used to estimate the rotational temperature and column density of excited $\mathrm{HCOOCH}_{3}$ using the rotational diagram method (Fig. 2, Table 4). Remijan et al. (2002) mapped W51 e2 with BIMA in two transitions of $\mathrm{HCOOCH}_{3}$ at $228.629 \mathrm{GHz}$ and $90.146 \mathrm{GHz}$. The size of the emission region in these lines was of about $7 "$ and $12^{\prime \prime}$, respectively. Adopting these values for torsionally excited $\mathrm{HCOOCH}_{3}$, we estimated a rotational temperature and a column density of $T_{\text {rot }}=131 \pm 20 \mathrm{~K}$ and $N=3.4_{-1.1}^{+1.5} \times 10^{16} \mathrm{~cm}^{-2}$, respectively, for a source size of $12^{\prime \prime}$, and $T_{\text {rot }}=104 \pm 14 \mathrm{~K}$ and $N=9.4_{-2.8}^{+4.0} \times 10^{16} \mathrm{~cm}^{-2}$, for a source size of $7^{\prime \prime}$ (Table 4). A separate analysis of the A and $\mathrm{E}$ lines of excited methyl formate provided compatible values, within the uncertainty, for the rotational temperature and column density. Transitions from the ground state of $\mathrm{HCOOCH}_{3}$ were also detected. Using the rotational diagram method, we measured for $\mathrm{HCOOCH}_{3} v_{\mathrm{t}}=0$, $T_{\text {rot }}=199 \pm 28 \mathrm{~K}$ and $N=9.6_{-0.9}^{+0.9} \times 10^{16} \mathrm{~cm}^{-2}$ for a $12^{\prime \prime}$ source (Fig. 3). If we consider both lines from the ground state and from $v_{\mathrm{t}}=1$, we find $T_{\text {rot }}=154 \pm 8 \mathrm{~K}$ and $N=5.6_{-3.7}^{+4.0} \times 10^{16} \mathrm{~cm}^{-2}$ for a $12^{\prime \prime}$ source (Fig. 3, Table 4). These temperatures and column densities were used to model the emission of methyl formate in the source. The comparison of the modeled spectra with observations is shown in Fig. 1 for a number of lines from $\mathrm{HCOOCH}_{3}$, $v_{\mathrm{t}}=1$.

\section{Ethyl cyanide}

Severals tens of lines from ethyl cyanide in the ground state are observed in the spectra. A large number of these lines are not blended and their energies cover a wide range, allowing us to plot a rotational diagram (Fig. 4). Adopting a source size of 12" as for the ground state of methyl formate, we found a rotational temperature of $T_{\text {rot }}=114 \pm 11 \mathrm{~K}$ and a column density $N=$ $1.7_{-0.2}^{+0.3} \times 10^{15} \mathrm{~cm}^{-2}$. Adopting a smaller source of $7^{\prime \prime}$ did not change the rotational temperature significantly but did alter the column density, such that $T_{\text {rot }}=103 \pm 9 \mathrm{~K}$ and a column density $N=3.7_{-0.5}^{+0.6} \times 10^{15} \mathrm{~cm}^{-2}$ (Table 4).

Ethyl cyanide appears to be colder than previously found. Liu et al. (2001) adopted a temperature of $200 \mathrm{~K}$ and measured an abundance for this molecule of $4 \times 10^{15} \mathrm{~cm}^{-2}$. However their analysis was based on lines of energies lower than $113 \mathrm{~K}$. If we limit ourselves to low energy transitions, we find $T_{\text {rot }}=184 \mathrm{~K}$ and a column density $N=1.3 \times 10^{16} \mathrm{~cm}^{-2}$. Ikeda et al. (2001) 
Table 1. Laboratory measurements, calculated frequencies and line strengths for methyformate transitions in the first torsionally excited state used in the present detection.

\begin{tabular}{|c|c|c|c|c|c|c|c|c|c|c|c|c|c|}
\hline$\overline{J^{\prime}}$ & $\begin{array}{c}K_{\mathrm{a}}^{\prime} \\
\text { Upper }\end{array}$ & $\begin{array}{c}K_{\mathrm{c}}^{\prime} \\
\operatorname{tate}^{a}\end{array}$ & $P^{\prime}$ & $J^{\prime \prime}$ & $\begin{array}{c}K_{\mathrm{a}}^{\prime \prime} \\
\text { Lowe }\end{array}$ & $\begin{array}{c}K_{\mathrm{c}}^{\prime \prime} \\
\text { state }^{a}\end{array}$ & $P^{\prime \prime}$ & $\begin{array}{l}\text { Obs. Freq. } \\
(\mathrm{MHz})^{b}\end{array}$ & $\begin{array}{l}\text { Calc. Freq. } \\
(\mathrm{MHz})^{c}\end{array}$ & $\begin{array}{c}\text { Calc. Unc } \\
(\mathrm{kHz})^{c}\end{array}$ & $\begin{array}{l}\text { Obs.-Calc. } \\
(\mathrm{MHz})^{c}\end{array}$ & $\begin{array}{l}S \mu^{2} \\
\left(\mathrm{D}^{2}\right)\end{array}$ & $\begin{array}{c}E_{\mathrm{l}} \\
\left(\mathrm{cm}^{-1}\right)^{d}\end{array}$ \\
\hline 7 & 4 & 4 & - & 6 & 4 & 3 & - & 85327.104 & 85327.085 & 11 & 0.019 & 12.483 & 146.8606 \\
\hline 7 & 4 & 3 & + & 6 & 4 & 2 & + & 85360.669 & 85360.823 & 11 & -0.154 & 12.483 & 146.8610 \\
\hline 7 & 3 & 5 & + & 6 & 3 & 4 & + & 85371.762 & 85371.789 & 11 & -0.027 & 15.120 & 143.6253 \\
\hline 7 & -6 & 2 & & 6 & -6 & 1 & & & 85456.630 & 19 & & 4.944 & 155.5393 \\
\hline 7 & 4 & 3 & & 6 & 4 & 2 & & 85506.175 & 85506.179 & 16 & -0.004 & 12.571 & 146.6097 \\
\hline 7 & -5 & 3 & & 6 & -5 & 2 & & 85553.365 & 85553.288 & 17 & 0.077 & 9.123 & 150.4139 \\
\hline 10 & 2 & 9 & - & 10 & 1 & 10 & + & 85664.038 & 85663.943 & 20 & 0.095 & 1.403 & 151.8343 \\
\hline 11 & 4 & 7 & + & 11 & 3 & 8 & - & 85727.753 & 85727.728 & 18 & 0.025 & 3.078 & 162.4132 \\
\hline 7 & -4 & 4 & & 6 & -4 & 3 & & 85743.967 & 85743.894 & 16 & 0.073 & 12.532 & 146.2356 \\
\hline 7 & 2 & 5 & + & 6 & 2 & 4 & + & 88998.368 & 88998.415 & 12 & -0.047 & 17.049 & 141.4958 \\
\hline 7 & 2 & 5 & & 6 & 2 & 4 & & 89140.383 & 89140.366 & 18 & 0.017 & 17.051 & 141.0232 \\
\hline 25 & 5 & 20 & - & 25 & 4 & 21 & + & & 107021.644 & 361 & & 8.289 & 274.8357 \\
\hline 9 & -2 & 8 & & 8 & -2 & 7 & & 107022.162 & 107022.092 & 13 & 0.070 & 22.628 & 146.6924 \\
\hline 10 & 1 & 9 & - & 9 & 2 & 8 & - & 107472.351 & 107472.387 & 15 & -0.036 & 2.144 & 150.7587 \\
\hline 9 & -3 & 7 & & 8 & -3 & 6 & & 111005.617 & 111005.582 & 19 & 0.035 & 20.800 & 149.1777 \\
\hline 9 & 1 & 8 & & 8 & 1 & 7 & & 111094.105 & 111094.056 & 13 & 0.049 & 23.222 & 146.0900 \\
\hline 12 & 0 & 12 & + & 11 & 0 & 11 & + & 131536.624 & 131536.653 & 12 & -0.029 & 31.375 & 155.8502 \\
\hline 12 & 0 & 12 & & 11 & 0 & 11 & & 131612.344 & 131612.301 & 13 & 0.043 & 31.558 & 155.3072 \\
\hline 12 & 1 & 12 & + & 11 & 0 & 11 & + & 131764.316 & 131764.341 & 12 & -0.025 & 4.870 & 155.8502 \\
\hline 11 & 5 & 7 & + & 10 & 5 & 6 & + & 134531.846 & 134531.833 & 13 & 0.013 & 23.125 & 164.8530 \\
\hline 27 & 7 & 20 & - & 27 & 6 & 21 & + & & 134545.609 & 582 & & 8.581 & 304.5077 \\
\hline 11 & -7 & 5 & & 10 & -7 & 4 & & 134545.615 & 134545.516 & 27 & 0.099 & 17.431 & 175.4588 \\
\hline 35 & 8 & 27 & + & 35 & 7 & 28 & - & & 134553.290 & 912 & & 12.622 & 416.6235 \\
\hline 11 & 5 & 6 & - & 10 & 5 & 5 & - & 134585.070 & 134585.141 & 13 & -0.071 & 23.125 & 164.8541 \\
\hline 11 & -3 & 9 & & 10 & -3 & 8 & & 134713.629 & 134713.526 & 16 & 0.103 & 26.708 & 156.9827 \\
\hline 11 & 5 & 6 & & 10 & 5 & 5 & & 134739.630 & 134739.629 & 19 & 0.001 & 23.251 & 164.7142 \\
\hline 12 & 2 & 11 & - & 11 & 2 & 10 & - & 140020.525 & 140020.564 & 12 & -0.039 & 30.616 & 158.9957 \\
\hline 11 & 2 & 9 & + & 10 & 2 & 8 & + & 140166.667 & 140166.713 & 12 & -0.046 & 28.103 & 155.9781 \\
\hline 12 & -2 & 11 & & 11 & -2 & 10 & & 140324.728 & 140324.699 & 12 & 0.029 & 30.787 & 158.5211 \\
\hline 13 & -1 & 13 & & 12 & -1 & 12 & & 142032.334 & 142032.293 & 14 & 0.041 & 34.222 & 159.7047 \\
\hline 13 & 0 & 13 & + & 12 & 0 & 12 & + & 142052.800 & 142052.735 & 14 & 0.065 & 34.029 & 160.2378 \\
\hline 13 & 0 & 13 & & 12 & 0 & 12 & & 142125.416 & 142125.410 & 14 & 0.006 & 34.224 & 159.6973 \\
\hline 13 & 1 & 13 & + & 12 & 0 & 12 & + & 142185.220 & 142185.198 & 14 & 0.022 & 5.339 & 160.2378 \\
\hline 15 & 0 & 15 & + & 14 & 1 & 14 & + & 163042.398 & 163042.248 & 20 & 0.100 & 6.267 & 170.0683 \\
\hline 15 & 1 & 15 & + & 14 & 1 & 14 & + & 163086.032 & 163085.873 & 20 & 0.159 & 39.344 & 170.0683 \\
\hline 15 & 0 & 15 & & 14 & -1 & 14 & & 163113.094 & 163113.156 & 18 & -0.062 & 6.073 & 169.5325 \\
\hline 15 & 0 & 15 & + & 14 & 0 & 14 & + & 163118.722 & 163118.574 & 20 & 0.148 & 39.345 & 170.0657 \\
\hline 14 & 1 & 13 & & 13 & 1 & 12 & & 163142.587 & 163142.607 & 14 & -0.020 & 36.185 & 168.1362 \\
\hline 15 & -1 & 15 & & 14 & -1 & 14 & & 163154.325 & 163154.404 & 18 & -0.079 & 39.561 & 169.5325 \\
\hline 15 & 0 & 15 & & 14 & 0 & 14 & & 163185.864 & 163185.922 & 18 & -0.058 & 39.561 & 169.5300 \\
\hline 15 & -1 & 15 & & 14 & 0 & 14 & & 163227.097 & 163227.169 & 18 & -0.072 & 6.074 & 169.5300 \\
\hline 16 & 0 & 16 & + & 15 & 1 & 15 & + & 173616.616 & 173616.407 & 25 & 0.209 & 6.727 & 175.5082 \\
\hline 16 & 1 & 16 & + & 15 & 1 & 15 & + & 173641.411 & 173641.171 & 25 & 0.240 & 42.007 & 175.5082 \\
\hline 16 & 0 & 16 & + & 15 & 0 & 15 & + & 173660.281 & 173660.032 & 25 & 0.249 & 42.007 & 175.5068 \\
\hline 16 & 0 & 16 & & 15 & -1 & 15 & & 173683.479 & 173683.597 & 23 & -0.118 & 6.525 & 174.9747 \\
\hline 24 & 3 & 21 & - & 24 & 2 & 22 & + & & 173704.470 & 241 & & 4.324 & 254.0651 \\
\hline 16 & -1 & 16 & & 15 & -1 & 15 & & 173706.683 & 173706.807 & 23 & -0.124 & 42.232 & 174.9747 \\
\hline 16 & 0 & 16 & & 15 & 0 & 15 & & 173724.731 & 173724.845 & 23 & -0.114 & 42.232 & 174.9733 \\
\hline 16 & -1 & 16 & & 15 & 0 & 15 & & 173747.990 & 173748.055 & 23 & -0.065 & 6.525 & 174.9733 \\
\hline 16 & 5 & 11 & & 15 & 5 & 10 & & 198384.885 & 198384.966 & 32 & -0.081 & 38.205 & 191.3885 \\
\hline 26 & 8 & 19 & - & 26 & 7 & 20 & + & & 198429.332 & 290 & & 6.921 & 297.4256 \\
\hline 16 & 5 & 11 & - & 15 & 5 & 10 & - & & 198539.350 & 30 & & 38.279 & 191.5148 \\
\hline 16 & -5 & 12 & & 15 & -5 & 11 & & 198578.563 & 198578.589 & 33 & -0.026 & 38.090 & 191.0593 \\
\hline 19 & 3 & 16 & - & 18 & 4 & 15 & - & & 207295.959 & 118 & & 2.804 & 208.1331 \\
\hline 17 & 12 & 5 & + & 16 & 12 & 4 & + & & 207354.058 & 101 & & 12.640 & 252.9573 \\
\hline 17 & 12 & 6 & - & 16 & 12 & 5 & - & & 207354.058 & 101 & & 12.640 & 252.9573 \\
\hline 17 & -13 & 5 & & 16 & -13 & 4 & & & 207376.777 & 145 & & 18.783 & 264.5661 \\
\hline 17 & 9 & 8 & & 16 & 9 & 7 & & & 207397.312 & 68 & & 32.511 & 223.9160 \\
\hline
\end{tabular}


Table 1. continued.

\begin{tabular}{|c|c|c|c|c|c|c|c|c|c|c|c|c|c|}
\hline$J^{\prime}$ & $\begin{array}{c}K_{\mathrm{a}}^{\prime} \\
\text { Upper }\end{array}$ & $\begin{array}{c}K_{\mathrm{c}}^{\prime} \\
\text { tate }^{a}\end{array}$ & $P^{\prime}$ & $J^{\prime \prime}$ & $\begin{array}{c}K_{\mathrm{a}}^{\prime \prime} \\
\text { Lowe }\end{array}$ & $\begin{array}{l}K_{\mathrm{c}}^{\prime \prime} \\
\text { state }^{a}\end{array}$ & $P^{\prime \prime}$ & $\begin{array}{l}\text { Obs. Freq. } \\
(\mathrm{MHz})^{b}\end{array}$ & $\begin{array}{l}\text { Calc. Freq. } \\
(\mathrm{MHz})^{c}\end{array}$ & $\begin{array}{l}\text { Calc. Unc } \\
(\mathrm{kHz})^{c}\end{array}$ & $\begin{array}{l}\text { Obs.-Calc. } \\
(\mathrm{MHz})^{c}\end{array}$ & $\begin{array}{l}S \mu^{2} \\
\left(\mathrm{D}^{2}\right)\end{array}$ & $\begin{array}{c}E_{\mathrm{l}} \\
\left(\mathrm{cm}^{-1}\right)^{d}\end{array}$ \\
\hline 17 & 11 & 7 & + & 16 & 11 & 6 & + & & 207404.993 & 81 & & 26.248 & 242.2767 \\
\hline 17 & 11 & 6 & - & 16 & 11 & 5 & - & & 207404.993 & 81 & & 26.248 & 242.2767 \\
\hline 17 & 10 & 8 & - & 16 & 10 & 7 & - & & 207500.297 & 66 & & 29.526 & 232.5324 \\
\hline 17 & 10 & 7 & + & 16 & 10 & 6 & + & & 207500.297 & 66 & & 29.526 & 232.5324 \\
\hline 19 & 2 & 18 & - & 18 & 1 & 17 & - & & 215130.469 & 47 & & 6.562 & 199.2115 \\
\hline 18 & 5 & 13 & - & 17 & 5 & 12 & - & & 225648.010 & 57 & & 44.072 & 205.2072 \\
\hline 19 & 2 & 17 & + & 18 & 2 & 16 & + & & 225702.569 & 46 & & 48.659 & 203.7419 \\
\hline 6 & 6 & 1 & - & 5 & 5 & 0 & - & & 225727.540 & 30 & & 2.592 & 148.5921 \\
\hline 6 & 6 & 0 & + & 5 & 5 & 1 & + & & 225727.552 & 30 & & 2.592 & 148.5921 \\
\hline 18 & 5 & 13 & & 17 & 5 & 12 & & & 225756.154 & 54 & & 43.428 & 205.0713 \\
\hline 7 & 6 & 1 & + & 6 & 5 & 2 & + & & 237899.077 & 27 & & 2.594 & 151.0254 \\
\hline 20 & 3 & 18 & + & 19 & 2 & 17 & + & & 237969.273 & 58 & & 5.371 & 211.2706 \\
\hline 37 & 7 & 31 & + & 37 & 6 & 32 & - & & 238027.934 & 1642 & & 8.537 & 434.5487 \\
\hline 20 & -14 & 7 & & 19 & -14 & 6 & & & 244000.464 & 195 & & 27.134 & 299.1664 \\
\hline 20 & 15 & 5 & - & 19 & 15 & 4 & - & & 244048.806 & 207 & & 23.017 & 312.5791 \\
\hline 20 & 15 & 6 & + & 19 & 15 & 5 & + & & 244048.806 & 207 & & 23.017 & 312.5791 \\
\hline 19 & 4 & 15 & + & 18 & 4 & 14 & + & & 244066.113 & 79 & & 48.355 & 209.5678 \\
\hline 27 & 1 & 26 & - & 27 & 0 & 27 & + & & 244066.575 & 470 & & 1.456 & 268.2307 \\
\hline 20 & 14 & 6 & + & 19 & 14 & 5 & + & & 244073.956 & 178 & & 20.522 & 299.0954 \\
\hline 20 & 14 & 7 & - & 19 & 14 & 6 & - & & 244073.956 & 178 & & 20.522 & 299.0954 \\
\hline 20 & 10 & 10 & & 19 & 10 & 9 & & & 244112.669 & 108 & & 39.852 & 254.7516 \\
\hline 20 & 13 & 7 & - & 19 & 13 & 6 & - & & 244119.960 & 154 & & 24.670 & 286.5471 \\
\hline 20 & 13 & 8 & + & 19 & 13 & 7 & + & & 244119.960 & 154 & & 24.670 & 286.5471 \\
\hline 20 & 12 & 8 & + & 19 & 12 & 7 & + & & 244198.512 & 134 & & 34.020 & 274.9352 \\
\hline 20 & 12 & 9 & - & 19 & 12 & 8 & - & & 244198.512 & 134 & & 34.020 & 274.9352 \\
\hline 20 & -13 & 8 & & 19 & -13 & 7 & & & 244207.619 & 159 & & 30.739 & 286.5459 \\
\hline
\end{tabular}

${ }^{a}$ Upper and lower state transitions quantum numbers. The rotational quantum number $J$, and the asymmetric rotor labels $K_{\mathrm{a}}$ and $K_{\mathrm{c}}$ are identified for each energy level. For the A symmetry species, $P$ is the parity quantum number, for the $E$ species, $P$ is not defined, instead, the $K_{\mathrm{a}}$ label has a signed value (Hougen et al. 1994; Ilyushin et al. 2008). Note that this information has been suppressed in Table 2, otherwise the quantum number labeling is the same. ${ }^{b}$ Observed laboratory frequencies in MHz from Ogata et al. (2004), the experimental uncertainty is $50 \mathrm{kHz}$ for all measured lines: ${ }^{c}$ calculated frequencies, uncertainties and obs-calc values are from (Carvajal et al. 2007) for the measured lines, the predicted frequencies and uncertainties for unmeasured lines is from Kleiner et al. (private communication); ${ }^{d}$ lower state energy in $\mathrm{cm}^{-1}$, relative to the $J=K=0 \mathrm{~A}$ species levels, set as zero energy.

Table 2. Detected transitions of the first torsionally excited state of $\mathrm{HCOOCH}_{3}$ in $\mathrm{W} 51$ e2.

\begin{tabular}{|c|c|c|c|c|c|c|c|c|}
\hline & 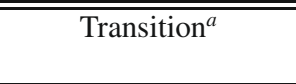 & $\begin{array}{l}S \mu^{2} \\
\left(\mathrm{D}^{2}\right)\end{array}$ & $\begin{array}{r}E_{1} \\
\left(\mathrm{~cm}^{-1}\right)\end{array}$ & $\begin{array}{r}\text { Frequency } \\
(\mathrm{MHz})\end{array}$ & $\begin{array}{r}\begin{array}{l}\text { Obs. Freq. } \\
(\mathrm{MHz})\end{array}\end{array}$ & $\begin{array}{c}\int_{\left(\mathrm{K} \mathrm{km} \mathrm{s}^{-1}\right)} T_{\mathrm{mb}} \Delta v^{c} \\
\mathrm{c}^{-1}\end{array}$ & $\begin{array}{r}T_{\mathrm{mb}} \\
(\mathrm{mK})\end{array}$ & " Comment $^{d}$ \\
\hline $1(8)$ & $7(4,4)-6(4,3) \mathrm{A}$ & 12.5 & 146.86 & 85327.104 & 85326.864 & 1.22 & 87 & \\
\hline $2(5)$ & $7(4,3)-6(4,2) \mathrm{A}$ & 12.5 & 146.86 & 85360.669 & 85359.685 & 0.40 & 81 & \\
\hline 3 & $7(3,5)-6(3,4) \mathrm{A}$ & 15.1 & 143.62 & 85371.762 & 85371.871 & 0.67 & 102 & \\
\hline 4 & $7(6,2)-6(6,1) \mathrm{E}$ & 4.94 & 155.53 & 85456.630 & 85456.067 & 14.33 & 1133 & $\mathrm{CH}_{3} \mathrm{CCH}$ \\
\hline $5(2)$ & $7(4,3)-6(4,2) E$ & 12.6 & 146.60 & 85506.175 & 85506.053 & 0.57 & 84 & \\
\hline 6 & $7(5,3)-6(5,2) \mathrm{E}$ & 9.12 & 150.40 & 85553.365 & 85553.361 & 0.54 & 78 & \\
\hline 7 & $10(2,9)-10(1,10) \mathrm{A}$ & 1.40 & 151.83 & 85664.038 & 85664.621 & 1.06 & 61 & $\mathrm{C}_{2} \mathrm{H}_{3} \mathrm{CN}$ \\
\hline $8(1)$ & $7(4,4)-6(4,3) \mathrm{E}$ & 12.5 & 146.22 & 85743.967 & 85743.915 & 0.79 & 102 & \\
\hline $9(10)$ & $7(2,5)-6(2,4) \mathrm{A}$ & 17.0 & 141.50 & 88998.368 & 88997.692 & 0.77 & 83 & \\
\hline $10(9)$ & $7(2,5)-6(2,4) \mathrm{E}$ & 17.1 & 141.01 & 89140.383 & 89140.161 & 1.20 & 120 & \\
\hline 11 & $25(5,20)-25(4,21) \mathrm{A}$ & 8.29 & 274.84 & 107021.644 & 107021.862 & 1.99 & 186 & \\
\hline 12 & $9(2,8)-8(2,7) \mathrm{E}$ & 22.6 & 146.68 & 107022.162 & - & - & - & \\
\hline 13 & $10(1,9)-9(2,8) \mathrm{A}$ & 2.14 & 150.76 & 107472.351 & 107472.400 & 0.27 & 45 & \\
\hline 14 & $9(3,7)-8(3,6) \mathrm{E}$ & 20.8 & 149.16 & 111005.617 & 111005.832 & 2.67 & 261 & \\
\hline 15 & $9(1,8)-8(1,7) \mathrm{E}$ & 23.2 & 146.08 & 111094.105 & 111094.010 & 2.27 & 244 & \\
\hline $16(17)$ & $12(0,12)-11(0,11) \mathrm{A}$ & 31.4 & 155.85 & 131536.624 & 131536.949 & 3.53 & 407 & \\
\hline $17(16)$ & $12(0,12)-11(0,11) \mathrm{E}$ & 31.6 & 155.29 & 131612.344 & 131612.094 & 3.97 & 410 & \\
\hline 18 & $12(1,12)-11(0,11) \mathrm{A}$ & 4.87 & 155.85 & 131764.316 & 131765.100 & 1.70 & 100 & $\mathrm{~S}^{18} \mathrm{O}$ \\
\hline 19 & $11(5,7)-10(5,6) \mathrm{A}$ & 23.1 & 164.85 & 134531.846 & 134531.411 & 3.44 & 478 & $\mathrm{SO}_{2} v_{2}=1$ \\
\hline 20 & $27(7,20)-27(6,21) \mathrm{A}$ & 8.58 & 304.51 & 134545.609 & 134546.431 & 7.95 & 630 & $\mathrm{C}_{3} \mathrm{H}_{2} \mathrm{CN}+{ }^{33} \mathrm{SO}_{2}$ \\
\hline 21 & $11(7,5)-10(7,4) E$ & 17.4 & 175.45 & 134545.615 & - & - & - & \\
\hline 22 & $35(8,27)-35(7,28) \mathrm{A}$ & 12.6 & 416.62 & 134553.290 & 134553.647 & 0.71 & 991 & ${ }^{33} \mathrm{SO}_{2}$ \\
\hline $23(25)$ & $11(5,6)-10(5,5) \mathrm{A}$ & 23.1 & 164.85 & 134585.070 & 134584.263 & 4.68 & 382 & $\mathrm{C}_{3} \mathrm{H}_{2} \mathrm{CN} v_{\mathrm{t}}, v_{\mathrm{b}}=1$ and? \\
\hline 24 & $11(3,9)-10(3,8) \mathrm{E}$ & 26.7 & 156.97 & 134713.629 & 134713.062 & 3.59 & 456 & \\
\hline $25(23)$ & $11(5,6)-10(5,5) \mathrm{E}$ & 23.3 & 164.70 & 134739.630 & 134739.110 & 3.55 & 386 & \\
\hline
\end{tabular}


Table 2. continued.

\begin{tabular}{|c|c|c|c|c|c|c|c|c|}
\hline & Transition $^{a}$ & $\begin{array}{l}S \mu^{2} \\
\left(\mathrm{D}^{2}\right)\end{array}$ & $\begin{array}{r}E_{1} \\
\left(\mathrm{~cm}^{-1}\right)\end{array}$ & $\begin{array}{r}\text { Frequency } \\
(\mathrm{MHz})\end{array}$ & $\begin{array}{r}\text { Obs. Freq. }{ }^{b} \\
(\mathrm{MHz})\end{array}$ & $\int_{\left(\mathrm{K} \mathrm{km} \mathrm{s}^{-1}\right)} T_{\mathrm{mb}} \Delta v^{c}$ & $\begin{array}{r}T_{\mathrm{mb}} \\
(\mathrm{mK})\end{array}$ & Comment $^{d}$ \\
\hline $26(28)$ & $12(2,11)-11(2,10) \mathrm{A}$ & 30.6 & 159.00 & 140020.525 & 140020.145 & 2.96 & 472 & \\
\hline 27 & $11(2,9)-10(2,8) \mathrm{A}$ & 28.1 & 155.98 & 140166.667 & 140166.502 & 3.42 & 528 & \\
\hline $28(26)$ & $12(2,11)-11(2,10) \mathrm{E}$ & 30.8 & 158.50 & 140324.728 & 140324.321 & 7.66 & 470 & \\
\hline 29 & $13(1,13)-12(1,12) \mathrm{E}$ & 34.2 & 159.69 & 142032.334 & 142031.811 & 4.52 & 586 & \\
\hline $30(31)$ & $13(0,13)-12(0,12) \mathrm{A}$ & 34.0 & 160.24 & 142052.800 & 142052.867 & 5.95 & 724 & \\
\hline $31(30)$ & $13(0,13)-12(0,12) \mathrm{E}$ & 34.2 & 159.68 & 142125.416 & 142125.086 & 4.01 & 559 & \\
\hline 32 & $13(1,13)-12(0,12) \mathrm{A}$ & 5.34 & 160.24 & 142185.220 & 142184.202 & 0.81 & 121 & \\
\hline $33(35)$ & $15(0,15)-14(1,14) \mathrm{A}$ & 6.27 & 170.07 & 163042.398 & 163042.050 & 2.32 & 363 & \\
\hline $34(38)$ & $15(1,15)-14(1,14) \mathrm{A}$ & 39.3 & 170.07 & 163086.032 & 163085.494 & 6.39 & 884 & $\mathrm{HCOOCH}_{3} v_{\mathrm{t}}=0$ \\
\hline $35(33)$ & $15(0,15)-14(1,14) \mathrm{E}$ & 6.07 & 169.52 & 163113.094 & 163113.656 & 5.11 & 549 & $\mathrm{SO}_{2}$ \\
\hline $36(39)$ & $15(0,15)-14(0,14) \mathrm{A}$ & 39.3 & 169.52 & 163118.722 & 163118.285 & 19.40 & 2184 & $\mathrm{SO}_{2}$ \\
\hline 37 & $14(1,13)-13(1,12) \mathrm{E}$ & 36.2 & 168.12 & 163142.587 & 163141.991 & 7.69 & 967 & \\
\hline $38(34)$ & $15(1,15)-14(1,14) \mathrm{E}$ & 39.6 & 162.52 & 163154.325 & 163153.604 & 8.99 & 855 & \\
\hline $39(36)$ & $15(0,15)-14(0,14) \mathrm{E}$ & 39.6 & 169.52 & 163185.864 & 163185.586 & 5.15 & 679 & \\
\hline 40 & $15(1,15)-14(0,14) \mathrm{E}$ & 6.07 & 169.52 & 163227.097 & 163227.290 & 4.23 & 448 & \\
\hline $41(44)$ & $16(0,16)-15(1,15) \mathrm{A}$ & 6.73 & 175.51 & 173616.616 & 173617.000 & 1.80 & 177 & \\
\hline $42(46)$ & $16(1,16)-15(1,15) \mathrm{A}$ & 42.0 & 175.51 & 173641.411 & 173636.978 & 15.14 & 1288 & $\mathrm{HCOOCH}_{3} v_{\mathrm{t}}=0$ \\
\hline $43(47)$ & $16(0,16)-15(0,15) \mathrm{A}$ & 42.0 & 175.51 & 173660.281 & 173659.384 & 4.12 & 380 & \\
\hline $44(41)$ & $16(0,16)-15(1,15) \mathrm{E}$ & 6.50 & 175.51 & 173683.479 & 173186.987 & 17.8 & 5300 & $\mathrm{CH}_{3} \mathrm{CHO}$ \\
\hline 45 & $24(3,21)-24(2,22) \mathrm{A}$ & 4.32 & 254.06 & 173704.470 & 173706.183 & 9.03 & 644 & \\
\hline $46(42)$ & $16(1,16)-15(1,15) \mathrm{E}$ & 42.2 & 174.96 & 173706.683 & 173706.183 & - & - & \\
\hline $47(43)$ & $16(0,16)-15(0,15) \mathrm{E}$ & 42.2 & 174.97 & 173724.731 & 173724.558 & 2.02 & 479 & \\
\hline 48 & $16(1,16)-15(0,15) \mathrm{E}$ & 6.6 & 174.97 & 173747.990 & 173748.150 & 3.94 & 264 & \\
\hline $49(51)$ & $16(5,11)-15(5,10) \mathrm{E}$ & 38.2 & 191.37 & 198384.885 & 198384.516 & 5.23 & 467 & \\
\hline 50 & $26(8,19)-26(7,20) \mathrm{A}$ & 6.92 & 297.43 & 198429.332 & 198428.202 & 1.67 & 231 & c- $\mathrm{C}_{2} \mathrm{H}_{4}$ \\
\hline $51(49)$ & $16(5,11)-15(5,10) \mathrm{A}$ & 38.3 & 191.52 & 198539.350 & 198541.500 & 19.44 & 1400 & $\mathrm{HCOOCH}_{3} v_{\mathrm{t}}=0$ \\
\hline 52 & $16(5,12)-15(5,11) \mathrm{E}$ & 38.1 & 191.05 & 198578.563 & 198577.928 & 3.20 & 484 & \\
\hline 53 & $19(3,16)-18(4,15) \mathrm{A}$ & 2.8 & 208.13 & 207295.959 & 207296.361 & 5.16 & 159 & $\mathrm{CH}_{3} \mathrm{CH}_{2} \mathrm{CN}$ \\
\hline 54 & $17(12,5)-16(12,4) \mathrm{A}$ & 21.2 & 252.96 & 207354.058 & 207352.197 & 5.57 & 557 & \\
\hline 55 & $17(12,6)-16(12,5) \mathrm{A}$ & 21.2 & 252.96 & - & - & - & - & \\
\hline 56 & $17(13,5)-16(13,4) \mathrm{E}$ & 18.8 & 264.55 & 207376.777 & 207378.506 & 11.46 & 995 & $\mathrm{CH}_{2} \mathrm{NH}$ \\
\hline 57 & $17(9,8)-16(9,7) \mathrm{E}$ & 32.5 & 223.90 & 207397.312 & 207396.134 & 8.62 & 758 & \\
\hline 58 & $17(11,6)-16(11,5) \mathrm{A}$ & 26.2 & 242.28 & 207404.993 & 207404.612 & 5.67 & 690 & \\
\hline 59 & $17(11,7)-16(11,6) \mathrm{A}$ & 26.2 & 242.28 & - & - & - & - & \\
\hline 60 & $17(10,8)-16(10,7) \mathrm{A}$ & 29.5 & 232.53 & 207500.297 & 207499.521 & 4.8 & 897 & \\
\hline 61 & $17(10,7)-16(10,6) \mathrm{A}$ & 29.5 & 232.53 & - & - & - & - & \\
\hline 62 & $19(2,18)-18(1,17) \mathrm{A}$ & 6.56 & 199.21 & 215130.469 & 215130.828 & 2.59 & 394 & $\mathrm{CH}_{3} \mathrm{CH}_{2} \mathrm{CN}$ \\
\hline $63(67)$ & $18(5,13)-17(5,12) \mathrm{A}$ & 44.1 & 205.21 & 225648.010 & 225647.974 & 5.16 & 1103 & $\mathrm{HCOOCH}_{3} v_{\mathrm{t}}=0$ \\
\hline 64 & $19(2,17)-18(2,16) \mathrm{A}$ & 48.7 & 203.74 & 225702.569 & 225698.559 & 184 & 1400 & $\mathrm{H}_{2} \mathrm{CO}$ \\
\hline 65 & $6(6,1)-5(5,0) \mathrm{A}$ & 2.59 & 148.59 & 225727.540 & 225726.732 & 2.55 & 372 & $\mathrm{CH}_{2} \mathrm{DCN}$ \\
\hline 66 & $6(6,0)-5(5,1) \mathrm{A}$ & 2.59 & 148.59 & 225727.552 & - & - & - & - \\
\hline $67(63)$ & $18(5,13)-17(5,12) \mathrm{E}$ & 43.4 & 205.06 & 225756.154 & 225755.178 & 7.3 & 927 & \\
\hline 68 & $7(6,1)-6(5,2) \mathrm{A}$ & 25.6 & 229.84 & 237899.08 & 237896.263 & 15.91 & 1.667 & $\mathrm{NH}_{2} \mathrm{CHO}$ \\
\hline 69 & $20(3,18)-19(2,17) \mathrm{A}$ & 5.37 & 211.27 & 237969.273 & 237969.938 & 12.80 & 1.186 & unidentified species \\
\hline 70 & $37(7,31)-37(6,32) \mathrm{A}$ & 8.54 & 434.55 & 238027.934 & 238026.742 & 1.12 & 122 & \\
\hline 71 & $20(14,7)-19(14,6) \mathrm{E}$ & 27.1 & 299.15 & 244000.464 & 243999.803 & 7.82 & 596 & \\
\hline 72 & $20(15,6)-19(15,5) \mathrm{A}$ & 23.3 & 312.58 & 244048.806 & 244048.230 & 90.33 & 10.84 & $\mathrm{H}_{2} \mathrm{CS}$ \\
\hline 73 & $20(15,5)-19(15,4) \mathrm{A}$ & 23.3 & 312.58 & - & - & - & - & - \\
\hline 74 & $19(4,15)-18(4,14) \mathrm{A}$ & 18.4 & 209.57 & 244066.113 & 244066.720 & 5.49 & 730 & \\
\hline 75 & $20(14,7)-19(14,6) \mathrm{A}$ & 14.2 & 299.10 & 244073.956 & 244073.440 & 3.54 & 314 & unidentified species \\
\hline 76 & $20(14,6)-19(14,5) \mathrm{A}$ & 14.2 & 299.10 & - & - & - & - & \\
\hline 77 & $20(10,10)-19(10,9) \mathrm{E}$ & 39.9 & 254.74 & 244112.669 & 244112.254 & 2.65 & 677 & \\
\hline $78(82)$ & $20(13,8)-19(13,7) \mathrm{A}$ & 21.0 & 286.55 & 244119.960 & 244119.708 & 2.95 & 886 & \\
\hline 79 & $20(13,7)-19(13,6) \mathrm{A}$ & 21.0 & 286.55 & - & - & - & - & \\
\hline 80 & $20(12,8)-19(12,7) \mathrm{A}$ & 34.0 & 274.94 & 244198.512 & 244198.232 & 7.43 & 1107 & \\
\hline 81 & $20(12,9)-19(12,8) \mathrm{A}$ & 34.0 & 274.94 & - & - & - & - & \\
\hline $82(78)$ & $20(13,8)-19(13,7) \mathrm{E}$ & 30.7 & 286.54 & 244207.619 & 244206.113 & 7.33 & 893 & unidentified species \\
\hline
\end{tabular}

${ }^{a}$ The notation for the quantum numbers is the same as Table 1 except that it does not contain information on the parity of the level, i.e. the signs present in Table 1 (associated to $K_{\mathrm{a}}$ for the $E$ species and in a separate column for the A species) have been suppressed; ${ }^{b}$ observed frequencies for a systemic velocity of $V_{\mathrm{lsr}}=55.3 \mathrm{~km} \mathrm{~s}^{-1}$; ${ }^{c}$ the line width is of the order of 7-8 $\mathrm{km} \mathrm{s}^{-1}$; ${ }^{d}$ this column indicates the molecules blended with the detected $\mathrm{HCOOCH}_{3} v_{\mathrm{t}}=1$ transitions. The dashes indicates that the value is the same as the one in the previous line. 

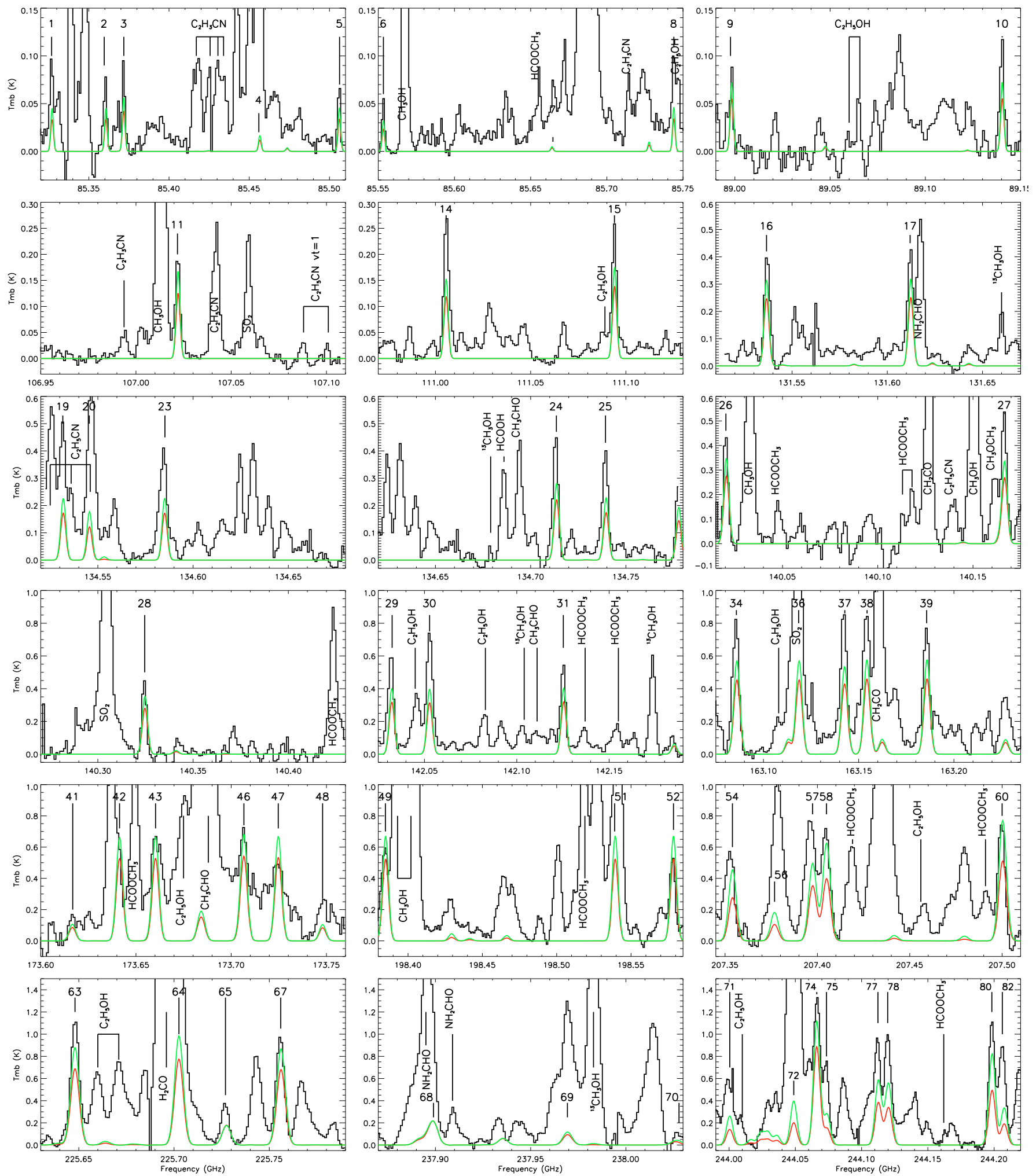

Fig. 1. Detected lines from the first torsionally excited state of methyl formate $\mathrm{HCOOCH}_{3}$. The observations (histogram like curve) are compared with LTE emission models of $\mathrm{HCOOCH}_{3} v_{\mathrm{t}}=1$ with $\theta_{\mathrm{s}}=7^{\prime \prime}, T_{\text {rot }}=104 \mathrm{~K}$ and $N=9.4 \times 10^{16} \mathrm{~cm}^{-2}$ (red curve) and with $\theta_{\mathrm{s}}=12^{\prime \prime}, T_{\text {rot }}=154 \mathrm{~K}$ and $N=5.6 \times 10^{16} \mathrm{~cm}^{-2}$ (green curve). The numbered lines are transitions from torsionally excited $\mathrm{HCOOCH}_{3}$, see Table 2 for the attribution of each line.

fixed $T_{\text {rot }}$ to be $150 \mathrm{~K}$ and found a column density of $N=7 \times$ $10^{14} \mathrm{~cm}^{-2}$.

We searched for transitions from the first excited bending mode (in-plane $\mathrm{CCN}$ bending mode) of ethyl cyanide at $206 \mathrm{~cm}^{-1}$ (designated by $v_{\mathrm{b}}=1$ ), and from the first torsional excited state, $v_{\mathrm{t}}=1$, at $212 \mathrm{~cm}^{-1}$. These 2 states and the CCN out-of-plane bending mode at $378 \mathrm{~cm}^{-1}$, were studied by Fukuyama et al. (1999) in the 8-200 GHz range for $J \leq 16$ and $K_{\mathrm{a}} \leq 2$. Lines from the two lowest energy states $v_{\mathrm{b}}=1$ and $v_{\mathrm{t}}=1$ were detected towards SgrB2 by Mehringer et al. (2004). 
Table 3. Detected transitions of excited $\mathrm{CH}_{3} \mathrm{CH}_{2} \mathrm{CN}\left(v_{\mathrm{b}}=1\right.$ and $\left.v_{\mathrm{t}}=1\right)$ in W51 e2.

\begin{tabular}{|c|c|c|c|c|c|c|c|c|}
\hline Transition & State & $\begin{array}{l}S \mu^{2} \\
\left(\mathrm{D}^{2}\right)\end{array}$ & $\begin{array}{r}E_{1} \\
\left(\mathrm{~cm}^{-1}\right)\end{array}$ & $\begin{array}{r}\text { Frequency } \\
(\mathrm{MHz})\end{array}$ & $\begin{array}{r}\text { Obs. Freq. }{ }^{a} \\
(\mathrm{MHz})\end{array}$ & $\begin{array}{c}\int_{\left(\mathrm{K} \mathrm{km} \mathrm{s}{ }^{-1}\right)} T_{\mathrm{mb}} \Delta v^{b} \\
\text {. }\end{array}$ & $\begin{array}{r}T_{\mathrm{mb}} \\
(\mathrm{mK})\end{array}$ & Comment \\
\hline $11_{8,4}-10_{8,3}$ & $v_{\mathrm{t}}=1 \mathrm{E}$ & 116.0 & 281.68 & 98463.46 & 98462.15 & 0.82 & 62 & \\
\hline $11_{8,3}-10_{8,2}$ & $v_{\mathrm{t}}=1 \mathrm{E}$ & - & - & 98464.02 & - & - & - & \\
\hline $11_{8,4}-10_{8,2}$ & $v_{\mathrm{t}}=1 \mathrm{~A}$ & - & - & 98464.27 & - & - & - & \\
\hline $11_{8,3}-10_{8,3}$ & $v_{\mathrm{t}}=1 \mathrm{~A}$ & - & - & 98464.32 & - & - & - & \\
\hline $11_{9,3}-10_{9,2}$ & $v_{\mathrm{t}}=1 \mathrm{E}$ & 79.2 & 295.40 & 98538.53 & 98537.9 & (c) & $\leq 15$ & $\mathrm{CH}_{3} \mathrm{CH}_{2} \mathrm{CN}$ \\
\hline $11_{9,2}-10_{9,1}$ & $v_{\mathrm{t}}=1 \mathrm{E}$ & - & - & 98538.84 & - & - & - & - \\
\hline $11_{9,3}-10_{9,1}$ & $v_{\mathrm{t}}=1 \mathrm{~A}$ & - & - & 98539.10 & - & - & - & - \\
\hline $11_{9,2}-10_{9,2}$ & $v_{\mathrm{t}}=1 \mathrm{~A}$ & - & - & 98539.11 & - & - & - & - \\
\hline $11_{7,4}-10_{7,3}$ & $v_{\mathrm{b}}=1 \mathrm{E}$ & 156.3 & 258.45 & 98556.61 & 98557.5 & (c) & $\leq 50$ & (c) \\
\hline $11_{7,5}-10_{7,4}$ & $v_{\mathrm{b}}=1 \mathrm{E}$ & - & - & 98556.88 & - & - & - & - \\
\hline $11_{7,5}-10_{7,3}$ & $v_{\mathrm{b}}=1 \mathrm{~A}$ & - & - & 98557.12 & - & - & - & - \\
\hline $11_{7,4}-10_{7,4}$ & $v_{\mathrm{b}}=1 \mathrm{~A}$ & - & - & 98557.27 & - & - & - & - \\
\hline $11_{6,6}-10_{6,5}$ & $v_{\mathrm{b}}=1 \mathrm{E}$ & 188.1 & 248.92 & 98617.87 & 98619.1 & 2.2 & 105 & (d) \\
\hline $11_{6,5}-10_{6,4}$ & $v_{\mathrm{b}}=1 \mathrm{E}$ & - & - & 98617.91 & - & - & - & - \\
\hline $11_{6,6}-10_{6,4}$ & $v_{\mathrm{b}}=1 \mathrm{~A}$ & - & - & 98618.09 & - & - & - & - \\
\hline $11_{6,5}-10_{6,5}$ & $v_{\mathrm{b}}=1 \mathrm{~A}$ & - & - & 98618.19 & - & - & - & - \\
\hline $11_{4,8}-10_{4,7}$ & $v_{\mathrm{t}}=1 \mathrm{~A}$ & 237.0 & 242.51 & 98619.45 & - & - & - & - \\
\hline $11_{5,7}-10_{5,5}$ & $v_{\mathrm{t}}=1 \mathrm{~A}$ & 199.0 & 249.94 & 98620.23 & - & - & - & - \\
\hline $11_{5,6}-10_{5,6}$ & $v_{\mathrm{t}}=1 \mathrm{~A}$ & - & - & 98620.33 & - & - & - & - \\
\hline $11_{4,8}-10_{4,7}$ & $v_{\mathrm{t}}=1 \mathrm{E}$ & 237.0 & 242.51 & 98620.38 & - & - & - & - \\
\hline $11_{5,7}-10_{5,6}$ & $v_{\mathrm{t}}=1 \mathrm{E}$ & 212.8 & 249.94 & 98620.41 & - & - & - & - \\
\hline $11_{4,7}-10_{4,6}$ & $v_{\mathrm{t}}=1 \mathrm{~A}$ & 237.0 & 242.51 & 98620.80 & - & - & - & - \\
\hline $11_{4,7}-10_{4,6}$ & $v_{\mathrm{t}}=1 \mathrm{E}$ & - & - & 98620.82 & - & - & - & - \\
\hline $11_{5,6}-10_{5,5}$ & $v_{\mathrm{t}}=1 \mathrm{E}$ & 212.8 & 249.94 & 98621.02 & - & - & - & - \\
\hline $11_{4,8}-10_{4,7}$ & $v_{\mathrm{b}}=1 \mathrm{~A}$ & 240.4 & 234.40 & 98735.64 & 98736.0 & 0.38 & 31 & \\
\hline $11_{4,7}-10_{4,6}$ & $v_{\mathrm{b}}=1 \mathrm{E}$ & 233.2 & - & 98735.77 & - & - & - & \\
\hline $11_{4,8}-10_{4,7}$ & $v_{\mathrm{b}}=1 \mathrm{E}$ & - & 234.40 & 98738.68 & 98738.8 & 0.57 & 75 & \\
\hline $11_{4,7}-10_{4,6}$ & $v_{\mathrm{b}}=1 \mathrm{~A}$ & 240.4 & - & 98739.06 & - & - & - & \\
\hline $12_{1,12}-11_{1,11}$ & $v_{\mathrm{b}}=1 \mathrm{E}$ & 297.2 & 229.03 & 107088.63 & 107088.5 & 0.52 & 50 & $\mathrm{C}_{2} \mathrm{H}_{5} \mathrm{OH}$ ? \\
\hline $12_{2,11}-11_{2,10}$ & $v_{\mathrm{b}}=1 \mathrm{~A}$ & - & 229.03 & 107088.81 & - & - & - & - \\
\hline $12_{2,11}-11_{2,10}$ & $v_{\mathrm{t}}=1 \mathrm{~A}$ & 294.5 & 235.76 & 107101.61 & 107101.2 & 0.34 & 37 & \\
\hline $12_{1,11}-11_{1,10}$ & $v_{\mathrm{t}}=1 \mathrm{E}$ & - & 235.76 & 107102.25 & - & - & - & \\
\hline $12_{8,4}-11_{8,3}$ & $v_{\mathrm{b}}=1 \mathrm{E}$ & 165.6 & 272.74 & 107251.81 & 107252.3 & 0.53 & 40 & \\
\hline $12_{8,5}-11_{8,4}$ & $v_{\mathrm{b}}=1 \mathrm{E}$ & - & - & 107252.50 & - & - & - & \\
\hline $12_{8,5}-11_{8,3}$ & $v_{\mathrm{b}}=1 \mathrm{~A}$ & - & - & 107252.56 & - & - & - & \\
\hline $12_{8,4}-11_{8,4}$ & $v_{\mathrm{b}}=1 \mathrm{~A}$ & - & - & 107252.93 & - & - & - & \\
\hline $12_{8,5}-11_{8,4}$ & $v_{\mathrm{t}}=1 \mathrm{E}$ & 148.1 & 284.97 & 107420.75 & 107420.9 & 0.80 & 40 & (d) \\
\hline $12_{8,4}-11_{8,3}$ & $v_{\mathrm{t}}=1 \mathrm{E}$ & - & - & 107421.34 & - & - & - & - \\
\hline $12_{8,5}-11_{8,3}$ & $v_{\mathrm{t}}=1 \mathrm{~A}$ & - & - & 107421.59 & - & - & - & - \\
\hline $12_{8,4}-11_{8,4}$ & $v_{\mathrm{t}}=1 \mathrm{~A}$ & - & - & 107421.66 & - & - & - & - \\
\hline $15_{4,12}-14_{4,11}$ & $v_{\mathrm{t}}=1 \mathrm{~A}$ & 333.4 & 257.47 & 134571.52 & 134571.2 & (c) & $\leq 10$ & (c) \\
\hline $15_{3,12}-14_{3,11}$ & $v_{\mathrm{t}}=1 \mathrm{E}$ & 329.8 & 257.47 & 134576.01 & - & - & - & - \\
\hline $15_{3,13}-14_{3,12}$ & $v_{\mathrm{t}}=1 \mathrm{~A}$ & 349.5 & 251.67 & 134589.47 & 134590.2 & 0.46 & 60 & $\mathrm{HCOOCH}_{3} v_{\mathrm{t}}=1$ \\
\hline $15_{2,13}-14_{2,12}$ & $v_{\mathrm{t}}=1 \mathrm{E}$ & - & - & 134590.45 & - & - & - & - \\
\hline $15_{6,10}-14_{6,9}$ & $v_{\mathrm{b}}=1 \mathrm{E}$ & 318.4 & 255.87 & 134616.20 & 134616.1 & 3.23 & 135 & (d) \\
\hline $15_{6,9}-14_{6,8}$ & $v_{\mathrm{b}}=1 \mathrm{E}$ & - & - & 134616.38 & - & - & - & - \\
\hline $15_{5,11}-14_{5,10}$ & $v_{\mathrm{b}}=1 \mathrm{~A}$ & 297.7 & 255.87 & 134616.44 & - & - & - & - \\
\hline $15_{5,10}-14_{5,9}$ & $v_{\mathrm{b}}=1 \mathrm{~A}$ & - & - & 134616.58 & - & - & - & - \\
\hline $15_{11,5}-14_{11,4}$ & $v_{\mathrm{b}}=1 \mathrm{E}$ & 140.1 & 326.77 & 134640.95 & 134640.4 & 1.93 & 257 & \\
\hline $15_{11,5}-14_{11,3}$ & $v_{\mathrm{b}}=1 \mathrm{~A}$ & - & - & 134641.10 & - & - & - & \\
\hline $15_{11,4}-14_{11,3}$ & $v_{\mathrm{b}}=1 \mathrm{E}$ & - & - & 134641.11 & - & - & - & \\
\hline $15_{11,4}-14_{11,4}$ & $v_{\mathrm{b}}=1 \mathrm{~A}$ & - & - & 134641.12 & - & - & - & \\
\hline $15_{12,4}-14_{12,3}$ & $v_{\mathrm{b}}=1 \mathrm{E}$ & 104.7 & 343.90 & 134648.79 & 134648.1 & 2.37 & 132 & (d) \\
\hline $15_{12,3}-14_{12,2}$ & $v_{\mathrm{b}}=1 \mathrm{E}$ & - & - & 134648.90 & - & - & - & - \\
\hline $15_{12,4}-14_{12,2}$ & $v_{\mathrm{b}}=1 \mathrm{~A}$ & - & - & 134648.98 & - & - & - & - \\
\hline $15_{12,3}-14_{12,3}$ & $v_{\mathrm{b}}=1 \mathrm{~A}$ & - & - & 134649.00 & - & - & - & - \\
\hline $15_{10,6}-14_{10,5}$ & $v_{\mathrm{b}}=1 \mathrm{E}$ & 175.0 & 311.15 & 134650.29 & - & - & - & - \\
\hline $15_{10,6}-14_{10,4}$ & $v_{\mathrm{b}}=1 \mathrm{~A}$ & - & - & 134650.34 & - & - & - & - \\
\hline $15_{10,5}-14_{10,5}$ & $v_{\mathrm{b}}=1 \mathrm{~A}$ & - & - & 134650.34 & - & - & - & - \\
\hline $15_{10,5}-14_{10,4}$ & $v_{\mathrm{b}}=1 \mathrm{E}$ & - & - & 134650.49 & - & - & - & - \\
\hline $15_{13,3}-14_{13,2}$ & $v_{\mathrm{b}}=1 \mathrm{E}$ & 69.2 & 362.56 & 134666.30 & 131667.6 & (c) & $\leq 10$ & (c) \\
\hline $15_{13,2}-14_{13,1}$ & $v_{\mathrm{b}}=1 \mathrm{E}$ & - & - & 134666.33 & - & - & - & - \\
\hline $15_{13,3}-14_{13,1}$ & $v_{\mathrm{b}}=1 \mathrm{~A}$ & - & - & 134666.49 & - & - & - & - \\
\hline $15_{13,2}-14_{13,2}$ & $v_{\mathrm{b}}=1 \mathrm{~A}$ & - & - & 134666.50 & - & - & - & - \\
\hline $15_{9,7}-14_{9,5}$ & $v_{\mathrm{b}}=1 \mathrm{~A}$ & 209.4 & 297.07 & 134703.14 & 134702.7 & 0.98 & 103 & \\
\hline
\end{tabular}


Table 3. continued.

\begin{tabular}{|c|c|c|c|c|c|c|c|c|}
\hline Transition & State & $\begin{array}{l}S \mu^{2} \\
\left(\mathrm{D}^{2}\right)\end{array}$ & $\begin{array}{r}E_{1} \\
\left(\mathrm{~cm}^{-1}\right)\end{array}$ & $\begin{array}{r}\text { Frequency } \\
(\mathrm{MHz})\end{array}$ & $\begin{array}{r}\text { Obs. Freq. }^{a} \\
(\mathrm{MHz})\end{array}$ & $\begin{array}{c}\int_{\left(\mathrm{K} \mathrm{km} \mathrm{s}^{-1}\right)} T_{\mathrm{mb}} \Delta v^{b} \\
\text {. }\end{array}$ & $\begin{array}{r}T_{\mathrm{mb}} \\
(\mathrm{mK})\end{array}$ & Comment \\
\hline $15_{9,6}-14_{9,6}$ & $v_{\mathrm{b}}=1 \mathrm{~A}$ & - & - & 134703.26 & - & - & - & \\
\hline $15_{9,7}-14_{9,6}$ & $v_{\mathrm{b}}=1 \mathrm{E}$ & - & - & 134703.45 & - & - & - & \\
\hline $15_{9,6}-14_{9,5}$ & $v_{\mathrm{b}}=1 \mathrm{E}$ & - & - & 134703.54 & - & - & - & \\
\hline $23_{0,23}-22_{0,22}$ & $v_{\mathrm{b}}=1 \mathrm{E}$ & 514.8 & 280.29 & 198463.40 & 198465.5 & 4.17 & 348 & unidentified species \\
\hline $23_{0,23}-22_{0,22}$ & $v_{\mathrm{b}}=1 \mathrm{~A}$ & - & - & 198463.42 & - & - & - & - \\
\hline $23_{4,19}-22_{4,18}$ & $v_{\mathrm{b}}=1 \mathrm{E}$ & 485.2 & 293.79 & 207396.15 & 207397.5 & 8.58 & 758 & $\mathrm{HCOOCH}_{3} v_{\mathrm{t}}=1$ \\
\hline $23_{4,20}-22_{4,19}$ & $v_{\mathrm{b}}=1 \mathrm{~A}$ & - & 293.79 & 207396.27 & - & - & - & - \\
\hline $23_{1,22}-22_{1,21}$ & $v_{\mathrm{t}}=1 \mathrm{~A}$ & 502.5 & 290.47 & 207415.76 & 207240.2 & 6.69 & 568 & $\mathrm{HCOOCH}_{3} v_{\mathrm{t}}=1$ \\
\hline $23_{1,23}-22_{1,22}$ & $v_{\mathrm{t}}=1 \mathrm{E}$ & - & 290.47 & 207417.59 & - & - & - & - \\
\hline $24_{0,24}-23_{1,23}$ & $v_{\mathrm{t}}=1 \mathrm{E}$ & 188.2 & 293.36 & 207446.75 & 207446.0 & (c) & $\leq 15$ & $\mathrm{C}_{2} \mathrm{H}_{5} \mathrm{OH}$ \\
\hline $24_{0,24}-23_{1,23}$ & $v_{\mathrm{b}}=1 \mathrm{~A}$ & 186.9 & 293.36 & 207447.53 & - & - & - & - \\
\hline $24_{11,14}-23_{11,13}$ & $v_{\mathrm{t}}=1 \mathrm{E}$ & 325.5 & 393.52 & 215133.09 & 215132.5 & (c) & $\leq 380$ & unknown species \\
\hline $24_{11,13}-23_{11,12}$ & $v_{\mathrm{t}}=1 \mathrm{E}$ & - & - & 215133.41 & - & - & - & - \\
\hline $24_{11,14}-23_{11,12}$ & $v_{\mathrm{t}}=1 \mathrm{~A}$ & - & - & 215134.13 & - & - & - & - \\
\hline $24_{11,13}-23_{11,13}$ & $v_{\mathrm{t}}=1 \mathrm{~A}$ & - & - & 215134.19 & - & - & - & - \\
\hline $25_{4,21}-24_{4,20}$ & $v_{\mathrm{b}}=1 \mathrm{E}$ & 511.8 & 307.94 & 225715.53 & 225714.7 & (c) & $\leq 228$ & (c) \\
\hline $25_{4,22}-24_{4,21}$ & $v_{\mathrm{b}}=1 \mathrm{~A}$ & - & - & 225715.61 & - & & & \\
\hline $27_{4,23}-26_{4,22}$ & $v_{\mathrm{b}}=1 \mathrm{E}$ & 534.4 & 323.30 & 244142.10 & 244139.3 & 3.94 & 288 & \\
\hline $27_{4,24}-26_{4,23}$ & $v_{\mathrm{b}}=1 \mathrm{~A}$ & - & - & 244142.13 & - & - & - & \\
\hline $28_{1,27}-27_{1,26}$ & $v_{\mathrm{t}}=1 \mathrm{~A}$ & 539.3 & 327.94 & 250318.38 & 250316.2 & 2.7 & 298 & \\
\hline $28_{2,27}-27_{2,26}$ & $v_{\mathrm{t}}=1 \mathrm{E}$ & 269.5 & 327.94 & 250319.81 & - & - & - & \\
\hline $28_{2,27}-27_{2,26}$ & $v_{\mathrm{t}}=1 \mathrm{E}$ & - & - & 250319.81 & - & - & - & \\
\hline $28_{15,13}-27_{15,12}$ & $v_{\mathrm{b}}=1 \mathrm{E}$ & 273.8 & 486.17 & 251372.86 & 251373.7 & (c) & $\leq 10$ & (c) \\
\hline $28_{15,14}-27_{15,13}$ & $v_{\mathrm{b}}=1 \mathrm{E}$ & - & - & 251373.06 & - & - & - & - \\
\hline $28_{15,14}-27_{15,12}$ & $v_{\mathrm{b}}=1 \mathrm{~A}$ & - & - & 251373.29 & - & - & - & - \\
\hline $28_{15,13}-27_{15,13}$ & $v_{\mathrm{b}}=1 \mathrm{~A}$ & - & - & 251373.32 & - & - & - & - \\
\hline
\end{tabular}

${ }^{a}$ Observed frequencies for a systemic velocity of $V_{\mathrm{lsr}}=57 \mathrm{~km} \mathrm{~s}^{-1} ;^{b}$ the line width is of the order of 7-8 $\mathrm{km} \mathrm{s}^{-1}{ }^{c}$ line within the noise, a Gaussian fit could not be made, constrains the abundance of excited ethyl cyanide; ${ }^{d}$ very broad lines, $\left(\Delta v \geq 15 \mathrm{~km} \mathrm{~s}^{-1}\right)$, due to the blending of several excited ethyl cyanide transitions and/or to the blending with an unknown species.

The dashes indicates that the value is the same as the one in the previous line.

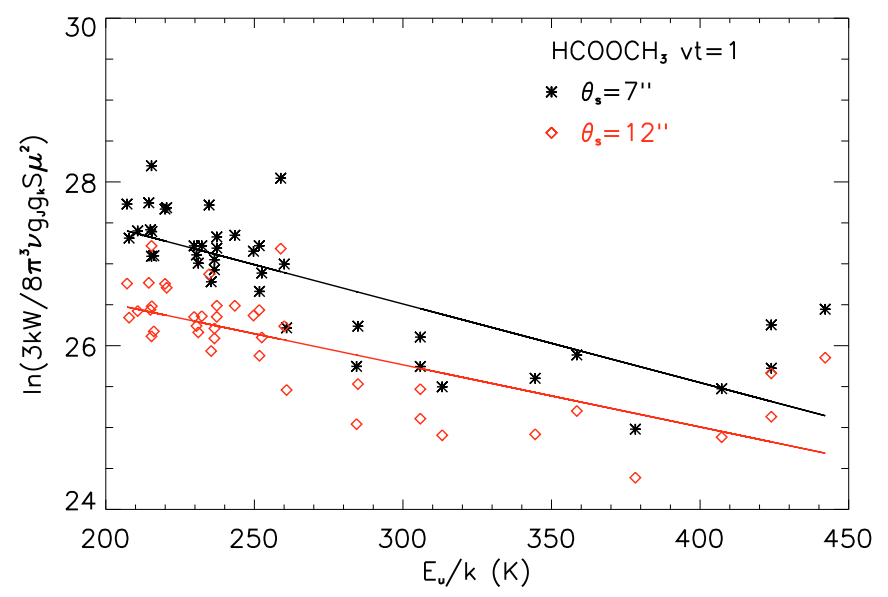

Fig. 2. Rotational diagram of the torsionally excited state of methyl formate for different source sizes. For a source size of 7" (black stars), we found a rotational temperature of $T_{\text {rot }}=104 \pm 14 \mathrm{~K}$ and a column density of $N=9.4_{-2 .}^{+4.0} \times 10^{16} \mathrm{~cm}^{-2}$; for a source size of $12^{\prime \prime}$ (red diamonds), we found $T_{\text {rot }}=131 \pm 20 \mathrm{~K}$ and $N=3.4_{-1.1}^{+1.5} \times 10^{16} \mathrm{~cm}^{-2}$.

This paper presents the molecular theory used for the spectral analysis of new measurements of excited ethyl cyanide in the 85-400 GHz range for $J \leq 50$ and $K_{\mathrm{a}} \leq 15$. However, the complete analysis has not yet been published and the prediction for the line frequencies and intensities was obtained from $J$. Pearson (private communication).

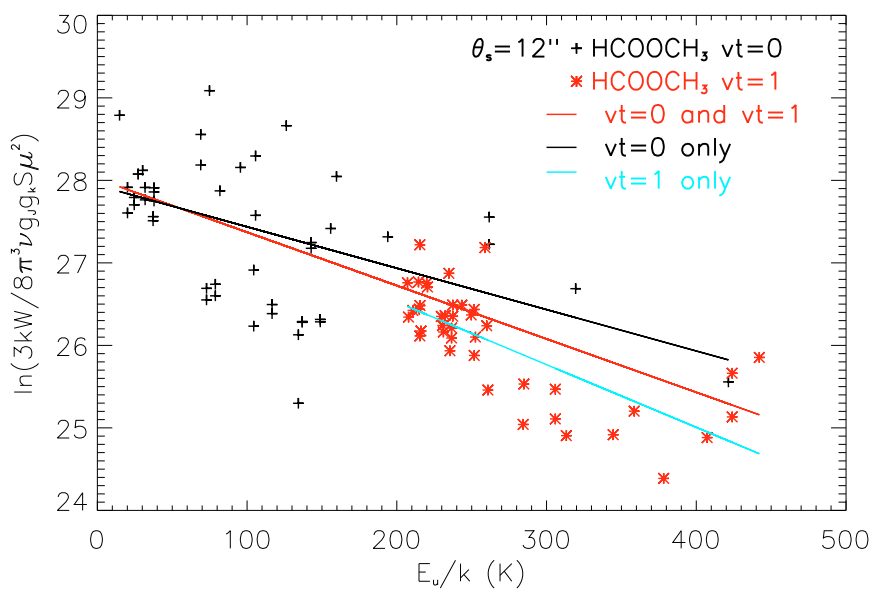

Fig. 3. Rotational diagram of methyl formate in the ground state (black crosses) and methyl formate in both the ground and excited states (red stars). The source size is $12^{\prime \prime}$. The rotational temperature and column density for methyl formate in the ground state are $T_{\text {rot }}=199 \pm 28 \mathrm{~K}$ and $N=9.6_{-0.9}^{+0.9} \times 10^{16} \mathrm{~cm}^{-2}$ and $T_{\text {rot }}=154 \pm 8 \mathrm{~K}$ and $N=5.6_{-3.7}^{+4.0} \times 10^{16} \mathrm{~cm}^{-2}$ when the ground and the first torsionally excited state are combined. For comparison, the rotational diagram for the excited state only is plotted (blue curve).

Most lines belonging to the excited states $v_{\mathrm{t}}=1$ and $v_{\mathrm{b}}=1$ are blended with other strong lines, identified or not (Table 3 ). However, few lines allow the column density of vibrationally excited ethyl cyanide to be constrained (Table 3). We estimated the upper limit to the abundance of excited $\mathrm{CH}_{3} \mathrm{CH}_{2} \mathrm{CN}$ by comparing the emission spectrum of excited ethyl cyanide simulated 


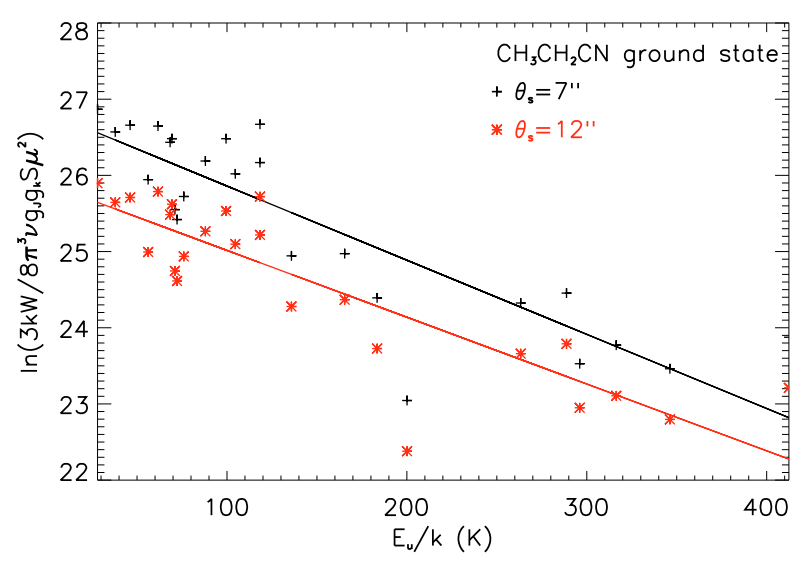

Fig. 4. Rotational diagram of the ethyl cyanide in the ground state for different source sizes. For a source size of 7" (black crosses) we find a rotational temperature of $T_{\text {rot }}=103 \pm 9 \mathrm{~K}$ and a column density of $N=3.7_{-0.5}^{+0.6} \times 10^{15} \mathrm{~cm}^{-2}$; for a source size of $12^{\prime \prime}$ (red stars) we find $T_{\text {rot }}=114 \pm 11 \mathrm{~K}$ and $N=1.3 \times 10^{16} \times 10^{15} \mathrm{~cm}^{-2}$.

and the LTE model with the observed spectra, for different temperatures and column densities (Fig. 5). Adopting the same temperature as the ground state $(100 \mathrm{~K})$ and a higher temperature of $200 \mathrm{~K}$, we found that the column density of vibrationally excited ethyl cyanide is $10^{16} \mathrm{~cm}^{-2}$ and $5 \times 10^{15} \mathrm{~cm}^{-2}$, respectively.

\section{Methyl carbamate and glycine}

The initial aim of the observations was to search for methyl carbamate $\left(\mathrm{NH}_{2} \mathrm{COOCH}_{3}\right)$, an isomer of glycine $\left(\mathrm{NH}_{2} \mathrm{CH}_{2} \mathrm{COOH}\right)$. Methyl carbamate has a strong dipole moment and is energetically more stable than glycine, two reasons that make it a good candidate for interstellar detection. The rotational spectrum of methyl carbamate in the A torsional substate was studied in the 8-240 GHz frequency range (Bakri et al. 2002; Ilyushin et al. 2006). This study was extended by Groner et al. (2007), who measured transitions of both $\mathrm{A}$ and $\mathrm{E}$ torsional substates up to $371 \mathrm{GHz}$. Our first analysis of the observations from 2003 was performed with a prediction of lines frequencies and intensities produced for the A-transitions from the work of Bakri et al. (2002). We then completed a revised analysis of our data from 2003 and 2006 with the predictions given by Groner et al. (2007).

Several tens of lines from methyl carbamate were searched in spectral regions chosen carefully to avoid or limit confusion with spectral lines from other molecules. However, we did not detect methyl carbamate in our data. Furthermore, most of the lines were fully blended and only a few lines allowed us to constrain an upper limit to the abundance of methyl carbamate in W51 e2. By comparing the simulated emission spectrum of methyl carbamate at different temperatures and source sizes with the observed spectrum (Fig. 6), we constrained the upper limit of methyl carbamate in this source. For a small emission region $\left(7^{\prime \prime}\right)$ and warm gas $(200 \mathrm{~K})$, we found that $N \leq 5 \times 10^{14} \mathrm{~cm}^{-2}$. This upper limit decreases as the rotational temperature and/or the size of the emission source decreases. We measured $N \leq 2 \times 10^{14} \mathrm{~cm}^{-2}$ for $T_{\text {rot }}=100 \mathrm{~K}$, and $\theta_{\mathrm{s}}=12^{\prime \prime}$, and $N \leq 8 \times 10^{13} \mathrm{~cm}^{-2}$ for $T_{\text {rot }}=50 \mathrm{~K}$ and $\theta_{\mathrm{s}}=30^{\prime \prime}$.

Numerous transitions from both conformers of glycine are within our observed spectral ranges. Glycine is not detected in the spectra. Almost all lines are blended and only a few can be used to estimate roughly the upper limit of glycine in W51 e2.
Table 4. Temperature and column density of the detected molecules.

\begin{tabular}{crcc}
\hline \hline Molecules & & \multicolumn{2}{c}{ Source size } \\
& & \multicolumn{1}{c}{$7^{\prime \prime}$} & \multicolumn{1}{c}{$12^{\prime \prime}$} \\
\hline $\mathrm{HCOOCH}_{3}$ & $T(\mathrm{~K})$ & $176 \pm 24$ & $199 \pm 28$ \\
$v_{\mathrm{t}}=0$ & $N\left(\mathrm{~cm}^{-2}\right)$ & $17.0_{-1.6}^{+1.8} \times 10^{16}$ & $9.6_{-0.9}^{+0.9} \times 10^{16}$ \\
$\mathrm{HCOOCH}_{3}$ & $\mathrm{~T}(\mathrm{~K})$ & $104 \pm 14$ & $131 \pm 20$ \\
$v_{\mathrm{t}}=1$ & $N\left(\mathrm{~cm}^{-2}\right)$ & $9.4_{-2.8}^{+4.0} \times 10^{16}$ & $3.4_{-1.1}^{+1.5} \times 10^{16}$ \\
$\mathrm{HCOOCH}_{3}$ & $T(\mathrm{~K})$ & $144 \pm 7$ & $154 \pm 8$ \\
$v_{\mathrm{t}}=0+v_{t}=1$ & $N\left(\mathrm{~cm}^{-2}\right)$ & $11.4_{-0.8}^{+0.9} \times 10^{16}$ & $5.6_{-3.7}^{+4.0} \times 10^{16}$ \\
$\mathrm{CH}_{3} \mathrm{CH}_{2} \mathrm{CN}$ & $T(\mathrm{~K})$ & $103 \pm 9$ & $114 \pm 11$ \\
& $N\left(\mathrm{~cm}^{-2}\right)$ & $3.7_{-0.5}^{+0.6} \times 10^{15}$ & $1.7_{-0.2}^{+0.3} \times 10^{15}$ \\
\hline
\end{tabular}

This upper limit varies by an order of magnitude depending on the source size and rotational temperature. Assuming a rotational temperature of $100 \mathrm{~K}$, the upper limit to the column density of glycine is $3 \times 10^{14} \mathrm{~cm}^{2}$ for a $7^{\prime \prime}$ source size, and $6 \times 10^{13} \mathrm{~cm}^{2}$ for a $30^{\prime \prime}$ source size.

\section{Discussion}

We have found that the rotational temperatures derived from our rotational diagram analysis differ from the kinetic temperature, $T_{\text {kin }}=153(21) \mathrm{K}$, derived by Remijan et al. (2004). The rotational temperature of ethyl cyanide, of between $103 \mathrm{~K}$ and $114 \mathrm{~K}$, is lower than $T_{\text {kin }}$, independent of the adopted source size. It is higher than $T_{\text {kin }}$ if we consider methyl formate in its ground state (176-199 K), lower if we consider only the excited state (104-131 K), and of the same order if we consider simultaneously the ground state and the first excited state of methyl formate (144-154 K). This appears to indicates that the LTE hypothesis is probably not fully valid. However, despite this possible departure from LTE, the rotational diagram method, which assumes implicitly that LTE has been reached and that all temperatures are equivalent (i.e. $T_{\text {exc }}=T_{\text {kin }}=T_{\text {rot }}=T_{\text {vib }}$ ), is the only method that can be used to estimate the excitation temperature since a statistical analysis is ruled out by the absence of known collision rates for the studied molecules.

The relevance of the LTE assumption within each rotational level may also be evaluated by comparing the cloud density to the critical density of each vibrational state. Collisional rates are not known for molecules as complex as methyl formate or ethyl cyanide. However, it is possible to estimate roughly the critical density for these molecules by adopting the value of methanol interacting with $\mathrm{H}_{2}$, of the order of a few $10^{-11} \mathrm{~cm}^{3} \mathrm{~s}^{-1}$ (Pottage et al. 2004), and by assuming that it is the same for the ground and vibrational excited states. The Einstein coefficients of the rotational transitions are in the range $10^{-5}-10^{-6} \mathrm{~s}^{-1}$. The critical density is therefore approximately $10^{5}-10^{6} \mathrm{~cm}^{-3}$, i.e. comparable to the hydrogen density in W51 e2, which is estimated to be $n_{\mathrm{H}}=5(2) \times 10^{5} \mathrm{~cm}^{-3}$ (Remijan et al. 2004). There is thus a competition between collisional and radiative excitation and it is clear that the levels are probably not all thermalized.

The excitation mechanism populating the observed excited state of methyl formate and ethyl cyanide may be inferred in a similar way. The Einstein coefficients for ro-vibrational transitions from the excited states to the ground state are of the order of a few $10^{-1} \mathrm{~s}^{-1}$, far higher than for rotational transitions within the excited state $\left(10^{-5}-10^{-6} \mathrm{~s}^{-1}\right)$. Adopting the same value for the collisional rate as before (a few $10^{-11} \mathrm{~cm}^{3} \mathrm{~s}^{-1}$ ), the density needed to thermalize the levels in the excited state by collisions must therefore be higher than the critical density, which is of the order of $10^{10} \mathrm{~cm}^{-3}$. The dust in W51 e2 has a temperature 

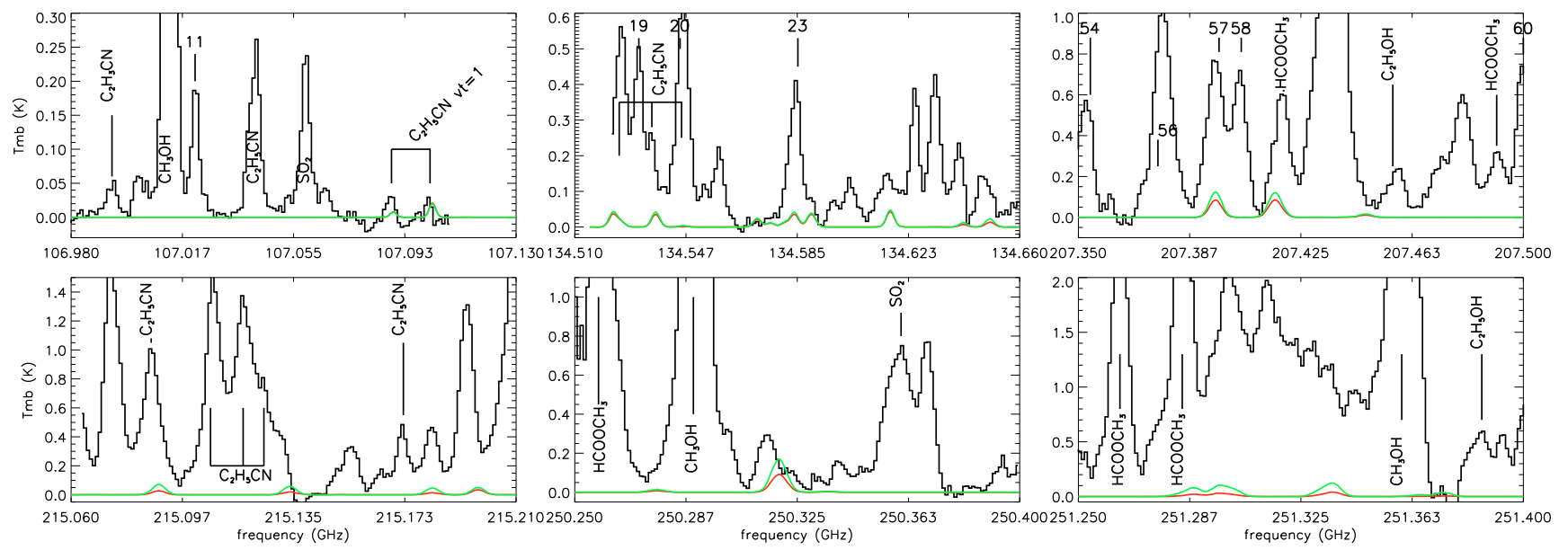

Fig. 5. Detected lines from the first two excited states $v_{\mathrm{t}}=1$ and $v_{\mathrm{b}}=1$ of ethyl cyanide $\mathrm{CH}_{3} \mathrm{CH}_{2} \mathrm{CN}$. The observations (histogram like curve) were compared with LTE emission models of $\mathrm{CH}_{3} \mathrm{CH}_{2} \mathrm{CN} v_{\mathrm{t}}=1$ and $v_{\mathrm{b}}=1$ with $\theta_{\mathrm{s}}=7^{\prime \prime}, T=100 \mathrm{~K}$ and $N=10^{16} \mathrm{~cm}^{-2}$ (red curve) and $T=200 \mathrm{~K}$ and $N=5 \times 10^{15} \mathrm{~cm}^{-2}$ (green curve). The numbered lines are excited methyl formate lines (see Table 2). See Table 3 for the identification of the excited $\mathrm{CH}_{3} \mathrm{CH}_{2} \mathrm{CN}$ lines.
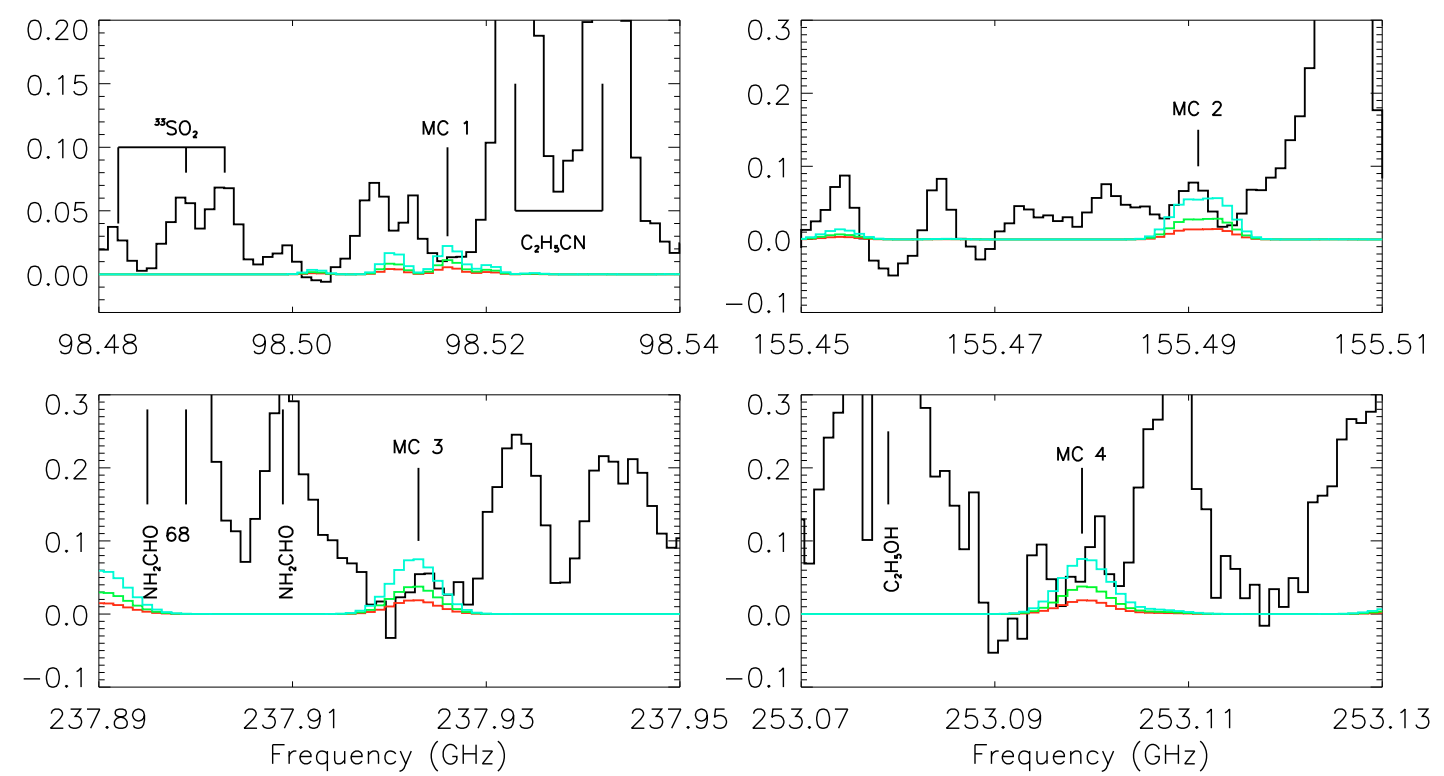

Fig. 6. Observed spectrum of W51 e2 (histogram like curve) compared with the LTE emission spectrum of methyl carbamate at different densities, for a $12^{\prime \prime}$ source and rotational temperature of $100 \mathrm{~K}$ : red curve: $N=10^{14} \mathrm{~cm}^{-2}$, green curve: $N=2 \times 10^{14} \mathrm{~cm}^{-2}$, blue curve: $N=4 \times 10^{14} \mathrm{~cm}^{-2}$. The methyl carbamate lines are (from Groner et al. 2007): MC1: $14_{2,13}-13_{1,12}$ (E) at $98515.38 \mathrm{GHz}$ and $15_{1,15}-14_{0,14} \mathrm{E}$ and A at $98516.99 \mathrm{GHz}$; MC2: $23_{1,22}-22_{2,21}$ at 155489.10 and $155492.68 \mathrm{GHz}$ for the $\mathrm{E}$ and A symmetry, respectively and $23_{2,22}-22_{1,21}$ at $155490.07 \mathrm{and} 155493.65 \mathrm{GHz}$ for the $\mathrm{E}$ and A symmetry, respectively; MC3 : $14_{10,5}-13_{9,4}$ (A) and $14_{10,4}-13_{9,5}$ (A) (blended) at $237922.60 \mathrm{GHz}$; MC4: $16_{10,7}-15_{9,6}(\mathrm{~A})$ and $16_{10,6}-15_{9,7}(\mathrm{~A})$ at 253099.30 and $253099.37 \mathrm{GHz}$, respectively.

of about $140 \mathrm{~K}$ (Sollins et al. 2004) and emits efficiently at the wavelength of the excited states of both molecules. It is thus most probable that the excited states of methyl formate and ethyl cyanide are populated by radiative processes rather than collisions.

We have found that the rotational temperature of the gas decreases as the source size becomes smaller. This is surprising since it is expected that the deepest regions of hot cores are also the warmest. The observed transitions are optically thin for the adopted source sizes of $12^{\prime \prime}$ and $7 "$. BIMA observations of methyl formate in W51 e2 (Remijan et al. 2002) showed that the emission region is smaller for high energy transitions than for low energy transitions. The size of the emission region was around $12^{\prime \prime}$ for the $7_{2,5}-6_{2,4}$ transition at $90146 \mathrm{MHz}$, corresponding to an energy of $10 \mathrm{~cm}^{-1}(15 \mathrm{~K})$, and around 7 " for the $18_{5,13}-17_{5,12}$ transition at $228629 \mathrm{MHz}$, corresponding to an energy of $75 \mathrm{~cm}^{-1}(108 \mathrm{~K})$. More interferometric observations are required to locate precisely the emission region of methyl formate and ethyl cyanide in the ground and excited state and understand their temperature distribution.

The presence of methyl formate in torsional excited state in hot cores such as W51 e2 and Orion KL (Kobayashi et al. 2007) is unsurprising. Methyl formate is abundant and its torsional mode has low energy $(188 \mathrm{~K})$. Similarly, it is unsurprising that ethyl cyanide is more difficult to detect: it is less abundant and its excited states are at slightly higher energy, around $300 \mathrm{~K}$. However, it is clear that observations of higher signal-tonoise ratio, spectral, and angular resolution, such as forthcoming 
observations from Herschel and ALMA, will reveal far more lines from the excited states of these molecules but also of abundant large molecules possessing low-frequency vibrational states. For example, dimethyl ether, $\mathrm{CH}_{3} \mathrm{OCH}_{3}$, has two torsional modes at about 203 and $242 \mathrm{~cm}^{-1}$ and transitions from these modes should be present in the spectra of hot cores. More generally, a large number of unidentified lines reported in the spectra of hot molecular clouds could be due to transitions from abundant molecules in excited states.

Acknowledgements. The authors would like to thank Alexandre Faure for fruitful discussions, Jean Demaison and Isabelle Kleiner for invaluable help in the methyl formate study and John Pearson for providing us with the excited ethyl cyanide spectrum. We acknowledge all the Pico Veleta IRAM staff for their help during the observations. We thank the referee for his/her constructive comments. This work was supported by the Programme National "Physico Chimie du Milieu Interstellaire" and by the European Research Training Network "Molecular Universe" (MRTN-CT-2004-512302). M.C. thanks the CNRS (project CERC3) and Junta de Andalucia (project P07-FQM-03014) for financial support.

\section{References}

Bakri, B., Demaison, J., Kleiner, I., et al. 2002, J. Mol. Spectrosc., 215, 312 Carvajal, M., Willaert, F., Demaison, J., \& Kleiner, I. 2007, J. Mol. Spectrosc., 246, 158

Demaison, J., Boucher, D., \& Dubrulle, A. 1984, J. Mol. Spectrosc., 102, 260

Fuchs, G. W., Fuchs, U., Giesen, T. F., \& Wyrowski, F. 2005, A\&A, 444, 521

Fukuyama, Y., Omori, K., Odashima, H., Takagi, K., \& Tsunekawa, S. 1999, J. Mol. Spectroscopy, 193, 72
Gordy, W., \& Cook, R. L. 1984, Microwave Molecular Spectra (New York: John Wiley \& Sons)

Groner, P., Winnewisser, M., Medvedev, I. R., et al. 2007, ApJS, 169, 28

Hollis, J. M., Lovas, F. J., Remijan, A. J., et al. 2006, ApJ, 643, L25

Hougen, J. T., Kleiner, I., \& Godefroid, M. 1994, J. Mol. Spectroscopy, 163, 559

Ikeda, M., Ohishi, M., Nummelin, A., et al. 2001, ApJ, 560, 792

Ilyushin, V., Alekseev, E., Demaison, J., \& Kleiner, I. 2006, J. Mol. Spectroscopy, 240, 127

Ilyushin, V., Kleiner, I., \& Lovas, F. J. 2008, J. Physical and Chemical Reference Data, 37, 97

Kleiner, I., Lovas, F. J., \& Godefroid, M. 1996, J. Physical and Chemical Reference Data, 25, 1113

Kobayashi, K., Ogata, K., Tsunekawa, S., \& Takano, S. 2007, ApJ, 657, L17

Liu, S.-Y., Mehringer, D. M., \& Snyder, L. E. 2001, ApJ, 552, 654

Mehringer, D. M., Pearson, J. C., Keene, J., \& Phillips, T. G. 2004, ApJ, 608, 306

Nummelin, A., Bergman, P., Hjalmarson, A., et al. 1998, ApJS, 117, 427

Oesterling, L. C., Albert, S., De Lucia, F. C., Sastry, K. V. L. N., \& Herbst, E. 1999, ApJ, 521, 255

Ogata, K., Odashima, H., Takagi, K., \& Tsunekawa, S. 2004, J. Mol. Spectroscopy, 225, 14

Plummer, G. M., Herbst, E., De Lucia, F., \& Blake, G. A. 1984, ApJS, 55, 633

Plummer, G. M., Herbst, E., De Lucia, F. C., \& Blake, G. A. 1986, ApJS, 60, 949

Pottage, J. T., Flower, D. R., \& Davis, S. L. 2004, MNRAS, 352, 39

Remijan, A., Snyder, L. E., Liu, S.-Y., Mehringer, D., \& Kuan, Y.-J. 2002, ApJ, 576,264

Remijan, A., Sutton, E. C., Snyder, L. E., et al. 2004, ApJ, 606, 917

Snyder, L. E., Lovas, F. J., Hollis, J. M., et al. 2005, ApJ, 619, 914

Sollins, P. K., Zhang, Q., \& Ho, P. T. P. 2004, ApJ, 606, 943

Turner, B. E. 1991, ApJS, 76, 617

Zhang, Q., Ho, P. T. P., \& Ohashi, N. 1998, ApJ, 494, 636 